\title{
The Enhancer of split and Achaete-Scute complexes of Drosophilids derived from simple ur-complexes preserved in mosquito and honeybee Rebekka Schlatter and Dieter Maier*
}

Address: Universität Hohenheim, Institut für Genetik, Garbenstr. 30, 70599 Stuttgart, GERMANY

Email: Rebekka Schlatter - rebekka.schlatter@gmx.de; Dieter Maier* - maierdie@uni-hohenheim.de

* Corresponding author

Published: 17 November 2005

BMC Evolutionary Biology 2005, 5:67 doi:10.1 |86/147|-2/48-5-67

This article is available from: http://www.biomedcentral.com/I47I-2/48/5/67

(c) 2005 Schlatter and Maier; licensee BioMed Central Ltd.

This is an Open Access article distributed under the terms of the Creative Commons Attribution License (http://creativecommons.org/licenses/by/2.0), which permits unrestricted use, distribution, and reproduction in any medium, provided the original work is properly cited.
Background: In Drosophila melanogaster the Enhancer of split-Complex [E(spl)-C] consists of seven highly related genes encoding basic helix-loop-helix (bHLH) repressors and intermingled, four genes that belong to the Bearded (Brd) family. Both gene classes are targets of the Notch signalling pathway. The Achaete-Scute-Complex [AS-C] comprises four genes encoding bHLH activators. The question arose how these complexes evolved with regard to gene number in the evolution of insects concentrating on Diptera and the Hymenoptera Apis mellifera.

Results: In Drosophilids both gene complexes are highly conserved, spanning roughly 40 million years of evolution. However, in species more diverged like Anopheles or Apis we find dramatic differences. Here, the $E(s p l)-C$ consists of one bHLH $(m \beta)$ and one Brd family member $(m \alpha)$ in a head to head arrangement. Interestingly in Apis but not in Anopheles, there are two more E(spl) bHLH like genes within $250 \mathrm{~kb}$, which may reflect duplication events in the honeybee that occurred independently of that in Diptera. The AS-C may have arisen from a single sc/l'sc like gene which is well conserved in Apis and Anopheles and a second ase like gene that is highly diverged, however, located within $50 \mathrm{~kb}$.

Conclusion: $E(s p l)-C$ and $A S-C$ presumably evolved by gene duplication to the nowadays complex composition in Drosophilids in order to govern the accurate expression patterns typical for these highly evolved insects. The ancestral ur-complexes, however, consisted most likely of just two genes: $E(s p l)-C$ contains one bHLH member of $m \beta$ type and one Brd family member of $m \alpha$ type and AS-C contains one scll'sc and a highly diverged ase like gene.

\section{Background}

The Notch pathway is one of the best studied cell to cell communication systems in the animal kingdom. It is highly conserved and used from worm to man. This pathway is needed whenever cell decisions are influenced by cell-cell communication, and also during proliferation or pathway crosstalk [1]. Using Drosophila melanogaster as a model system, the Notch pathway was intensely studied over many years. The best defined process governed by Notch is called "lateral inhibition": cells of a given fate are singled out from an equivalence group of the same fate, whereas the differentiation of the other cells is suppressed by the Notch signal. This happens for example during neurogenesis, where neuroblasts are selected from prone- 
A
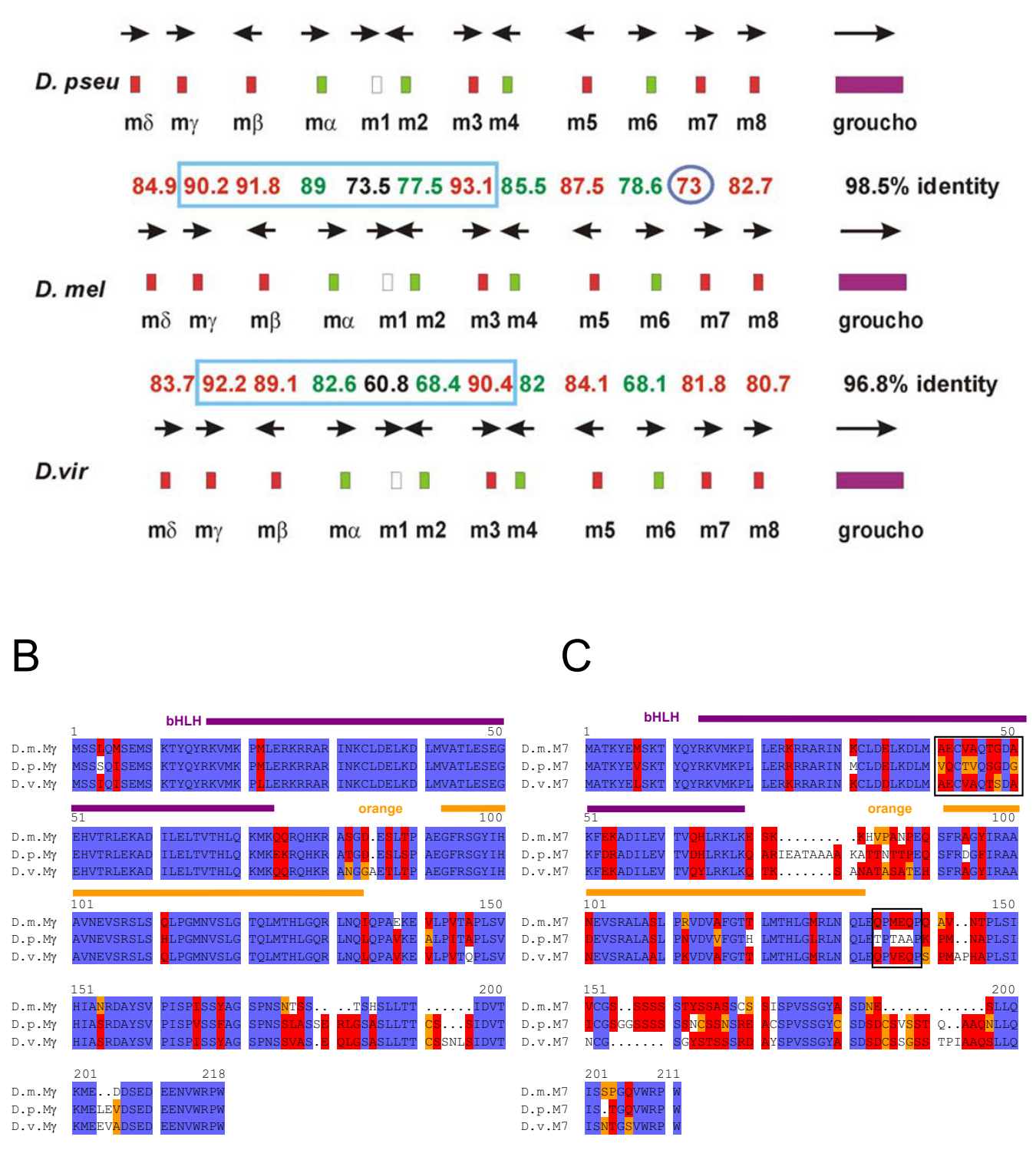

Figure I

Conservation of the $E(s p l)-C$ in Drosophilids. A) The $E(s p l)-C$ is highly conserved in Drosophilids with regard to gene number and transcript orientation (arrows). The size of the complex is also almost the same; in $D$. pseudoobscura (D. pseu) it is only slightly larger than in $D$. melanogaster (D. mel). The smallest seems to be the $D$. virilis $(D$. vir) complex, however, the virilis sequence was not completed at the time. The best identity at protein level is found between the Gro orthologs (purple) followed by the bHLH proteins (red). Interestingly the proteins of the centrally located bHLH genes M $\gamma, M \beta$ and M3 (framed blue) are best conserved. Higher identities are seen between the melanogaster and pseudoobscura orthologs than between the ones of $D$. melanogaster and $D$. virilis, with the exception of M7 (blue circle). The worst conserved member of the complex is MI. (Numbers give \% identity between the proteins). B) Alignment of the M $\gamma$ and (C) M7 orthologs. The bHLH (purple) and orange domains are the best conserved parts of the orthologs. The M7 sequences labelled with the black box are unexpectedly better conserved in $D$. virilis than in $D$. pseudoobscura compared with $D$. melanogaster. Identical residues are marked in blue; red shows highly related and yellow similar residues. 
ural clusters; they keep neural fate, whereas the surrounding cells eventually differentiate as epidermoblasts. The name giving transmembrane Notch-receptor interacts physically with the extracellular domain of the transmembrane ligands Delta or Serrate of the signalling cell. After this activation, the intracellular domain of Notch is cleaved and travels into the nucleus where it transcriptionally activates together with Suppressor of Hairless $[\mathrm{Su}(\mathrm{H})]$ genes of the Enhancer of split complex $[E(s p l)-C] . E(s p l)$ gene products in turn repress the activity of proneural genes encoded for example by the Achaete-Scute-Complex $[A S-C]$. As consequence these cells stay undifferentiated to become epidermoblasts later on, whereas the signalling cell enters into the programmed neural cell fate. In the focus of our studies are these two complexes, $E(s p l)-C$ and $A S-C$, since in D. melanogaster they are composed of several genes with complex expression patterns and specific yet partly redundant functions.

Enhancer of split was originally identified by genetic means as enhancer of the duplicated bristle phenotype found in the recessive Notch allele split [2]. In order to identify the responsible gene the Enhancer of split gene region has been cloned. In this region 13 transcription units are located and named $m 1$ to $m 10$ and $m \alpha$ to $m \delta$. It was a surprise that seven of these genes encode structurally related proteins characterized by a basic and a helix-loop-helix domain (bHLH), a further alpha-helix forming 'orange domain' and a stereotypic terminus with the amino acids tryptophane, arginine, proline, tryptophane (WRPW). Later it was shown that this motif serves as binding site for the global co-repressor Groucho (Gro, transcription unit m9/ $10)$, which is encoded by a gene localised next to the bHLH gene cluster [3-10]. The bHLH genes $m 3, m 5, m 7$, $m 8, m \beta, m \gamma$ and $m \delta$ (see Fig. $1 ;[8]$ ) are all transcriptional targets of Notch: they encode the effector proteins of the Notch signal at least in the process of lateral inhibition [1,11-13]. Apart from the seven bHLH genes and the neighbouring gro locus, the $E(s p l)-C$ comprises five further genes. Four genes $m \alpha, m 2, m 4$ and $m 6$ share structural similarity with the Bearded ( $\mathrm{Brd}$ )-gene family and are themselves transcriptional targets of Notch, whereas $m 1$ is completely unrelated and encodes a putative protease inhibitor $[14,15]$. Larger deficiencies encompassing several of $E(s p l)$ transcripts cause a severe neural hyperplasia, whereas loss of activity of single genes do not, suggesting redundancy of these seven bHLH genes $[7,10,16-18]$. However, remarkable differences were observed between the respective expression patterns in the embryo as well as in postembryonic tissues, arguing against complete redundancy $[8,12,14,15,19-22]$. Consistently, a high conservation of the entire complex was observed in the rather distantly related fly species Drosophila hydei [23]. The question, however, remains whether gene number and structure of the complex is conserved during longer terms of evolution. For example, in vertebrates an $E(s p l)-C$ like in D. melanogaster does not exist. Here the Notch target genes have been classified as HES (hairy/Enhancer of split) and HER/HESR (hairy/Enhancer of split related) genes, because the D. melanogaster segmentation gene hairy encodes a bHLH protein with orange domain and WRPW motif that is as similar to the vertebrate HES genes as are the $E(s p l)$ bHLH genes $[24,25]$. The vertebrate genes are not clustered in a complex. Apparently, in the course of evolution rearrangements occurred between these Notch target genes.

The AS-C in D. melanogaster comprises four genes, achaete $(a c)$, scute (sc), lethal of scute (l'sc) and asense (ase) that all encode transcriptional activators of the bHLH class. They determine proneural fate and are thus required for the development of the central and peripheral nervous system $[13,26-29]$. These genes have been also studied in vertebrates. In the mouse there are three achaete-scute family members abbreviated ASH for Ac-Sc-Homolog [30]: MASH- 1 and XASH-3/CASH- 4 are two members that are involved in the development of the nervous system [30]. Both complexes are therefore good candidates to look for the changes that occurred during insect evolution.

In this work, we studied the evolution of the $E(s p l)-C$ and $A S-C$ by making use of the recent advances in the genome projects of the Diptera D. pseudoobscura, D. virilis, Anopheles gambiae and the Hymenoptera Apis mellifera. The estimated distances are nearly 30 million years (Myr) separating D. melanogaster and D. pseudoobscura which both belong to the Sophophora subgenus and around 40 Myr separating the Sophophora from the Drosophila subgenus where D. virilis belongs to [31]. The distance between these modern dipterans and the more ancient ones like the Culicidae A. gambiae is estimated 200-250 Myr and that between Diptera and Hymenoptera like the honeybee A. mellifera 250-300 Myr [32]. Our study shows that both gene complexes are highly conserved in Drosophilids with regard to overall size, gene number and structural similarity of the encoded proteins. In contrast, more ancient dipterans like the mosquito and similarly also the honeybee have much simpler gene complex structures: the $E(s p l)-C$ consists of just one $m \beta$ like bHLH gene and one $m \alpha$ like Brd-type gene. However, in Apis two more bHLH/WRPW coding genes are found within about 200 $\mathrm{kb}$ and may reflect an enlargement of the $E(s p l)-C$ in this species. The AS-C consists of only one $s c / l$ 'sc like member and one further ase-like gene that is, however, widely diverged. These data suggest that the evolution of modern Drosophilids included an enlargement of these complexes, notably a multiplication of the genes in the $E(s p l)$ $C$ that seem to have subsequently specified their roles in Notch signalling pathway. Their strict conservation in Drosophilids argues for a diversification presumably 
driven by their highly specified expression patterns and regulatory activities.

\section{Results \\ The Enhancer of split complex in Drosophilids}

The Enhancer of split complex [E(spl)-C] consists of 13 transcription units (Fig. 1): seven genes $(m 3, m 5, m 7, m 8$, $m \beta, m \gamma, m \delta$ ) encode highly related basic helix-loop-helix proteins, four have been grouped to the Brd-family ( $m 2$, $m 4, m 6, m \alpha) ; m 1$ which encodes a serine protease inhibitor and l(3) groucho (gro) which encodes a co-repressor of the $\mathrm{E}(\mathrm{spl}) \mathrm{bHLH}$ protein family. Although the $\mathrm{E}(\mathrm{spl})$ bHLH proteins are partly redundant and, therefore, a loss or addition of genes could be without consequences, the complex is highly conserved in all studied Drosophilids with respect to gene order and number, transcription orientation and overall size. As expected, the evolution rate of the orthologs is different. The best conservation is found between the Gro orthologs (more than 96\% identity, Fig. 1), whereas M1 displays the highest evolutionary rate (less than $61 \%$ identity between $D$. melanogaster and D. virilis; Fig. 1).

\section{The $b H L H$ proteins of the $\mathrm{E}(\mathrm{spl})-\mathrm{C}$}

In the Drosophilids, all E(spl) bHLH genes are without intron, and the proteins contain the typical bHLH and orange domains and end with the WRPW motif. The best conserved bHLH ortholog is $\mathrm{M} \gamma$ followed by M3, M $\beta, \mathrm{M} 5$, $M \delta$ and M7/8 (Fig. 1). However, the evolutionary rate varies quite strongly. Comparing D. melanogaster with $D$. virilis, the highest identity score is found for $\mathrm{M} \gamma$ with $~ 92 \%$ (Fig. 1A,B) and the lowest for M8 with $\sim 81 \%$. An even more striking difference in the identity scores is observed when comparing the D. melanogaster and D. pseudoobscura orthologs M3 and M7 (93\% versus 73\%; Fig. 1A,C). The low degree of conservation of the M7 proteins is rather surprising. It is based on one hand on peculiar size variations: 206 residues in D. pseudoobscura, 186 in D. melanogaster and 197 in D. virilis (Fig. 1C). On the other hand, the amino acid composition of the bHLH and orange domains is much better conserved between D. melanogaster and $D$. virilis than between $D$. melanogaster and $D$. pseudoobscura. This is different from all the other bHLH orthologs: the bHLH domains of $\mathrm{M} 3$ and $\mathrm{M} \beta$ are identical in all three species and also the other ones are extremely similar. Only one conservative change is detected in the $\mathrm{M} \gamma$ bHLH domain of $D$. melanogaster compared with $D$. virilis, and just two in M5. The bHLH domains of the M8 and $M \delta$ proteins contain also single non-conservative replacements, apart from a few conservative changes. However, D. pseudoobscura M7 shows an unusual high number of changes - six replacements and five conservative changes - within the bHLH domain if compared to the melanogaster ortholog (Fig. 1C).

\section{The Bearded-protein family in the E(spl)-C}

In general, the Brd-type proteins evolve faster than the bHLH proteins: $M 4$ and $M \alpha$ are the best conserved members with $\sim 82 \%$ identity between virilis and melanogaster and are thus within the range of the fastest evolving $m 7 / 8$ bHLH coding genes (Fig. 1). The overall structure of M4 and $M \alpha$ orthologs is quite similar in all studied Drosophila species. The so-called 'bearded'-domain is completely identical in the $M \alpha$ orthologs, M4 has only a few gaps. However, the predicted $D$. virilis $m 4$ gene has an extended open reading frame of novel 132 residues at the 5' end, whereas the remaining 156 residues are conserved. The melanogaster $m 45^{\prime}$ region reveals similarity at the DNA level, however, has no open reading frame. Therefore it remains questionable whether the larger open reading frame in D. virilis is indeed translated. The other two Brdfamily members, M2 and M6, are much less conserved: Only approximately $68 \%$ identity is found between the respective orthologs of $D$. melanogaster and $D$. virilis. Despite this little conservation, the typical Brd protein domains can still be recognized. The most prominent is the predicted basic amphipathic $\alpha$-helix domain in the $\mathrm{N}$ terminal protein region [14].

\section{The $\mathrm{ml}$ gene in the $\mathrm{E}(\mathrm{spl})-\mathrm{C}$}

The $m 1$ gene encodes a protein that has the signatures of serine protease inhibitors [15]. Despite a low degree of conservation which ranges between 60 and $70 \%$ identity between the M1 orthologs (Fig. 1A), the structurally important cysteines residues are conserved in number and spacing [15]. Notably, the orthologous genes of D. pseudoobscura and $D$. virilis have a significantly longer open reading frame at the 5 'end that extends the proteins for approximately 50 residues to 203 in the case of $D$. virilis. The extended protein parts share $68 \%$ similarities within the first 30 residues between the two orthologs. Furthermore, the first nine residues have only one conservative exchange arguing for its translation in vivo. In melanogaster all three reading frames at the 5 ' end are blocked by several stop codons, excluding a likewise 5 ' extension. However, at the DNA level there are identities of $69 \%$ to the virilis ortholog and $79 \%$ to the pseudoobscura ortholog which could be also interpreted as conserved regulatory sequence.

\section{The E(spl)-C in Anopheles gambiae}

Albeit Anopheles belongs to the dipteran flies it does not contain an $E(s p l)-C$ that matches that of Drosophilids (Fig. 2A). Only a single transcription unit with respectable conservation that contains one intron was annotated in the genome project (ENSANGG00000017601; see Tab. $1)$. However, the predicted coding sequence does not end with a WRPW motif as expected for $\mathrm{E}$ (spl) bHLH proteins. By searching through the genomic sequence, we propose a different gene structure, where the transcript extends 

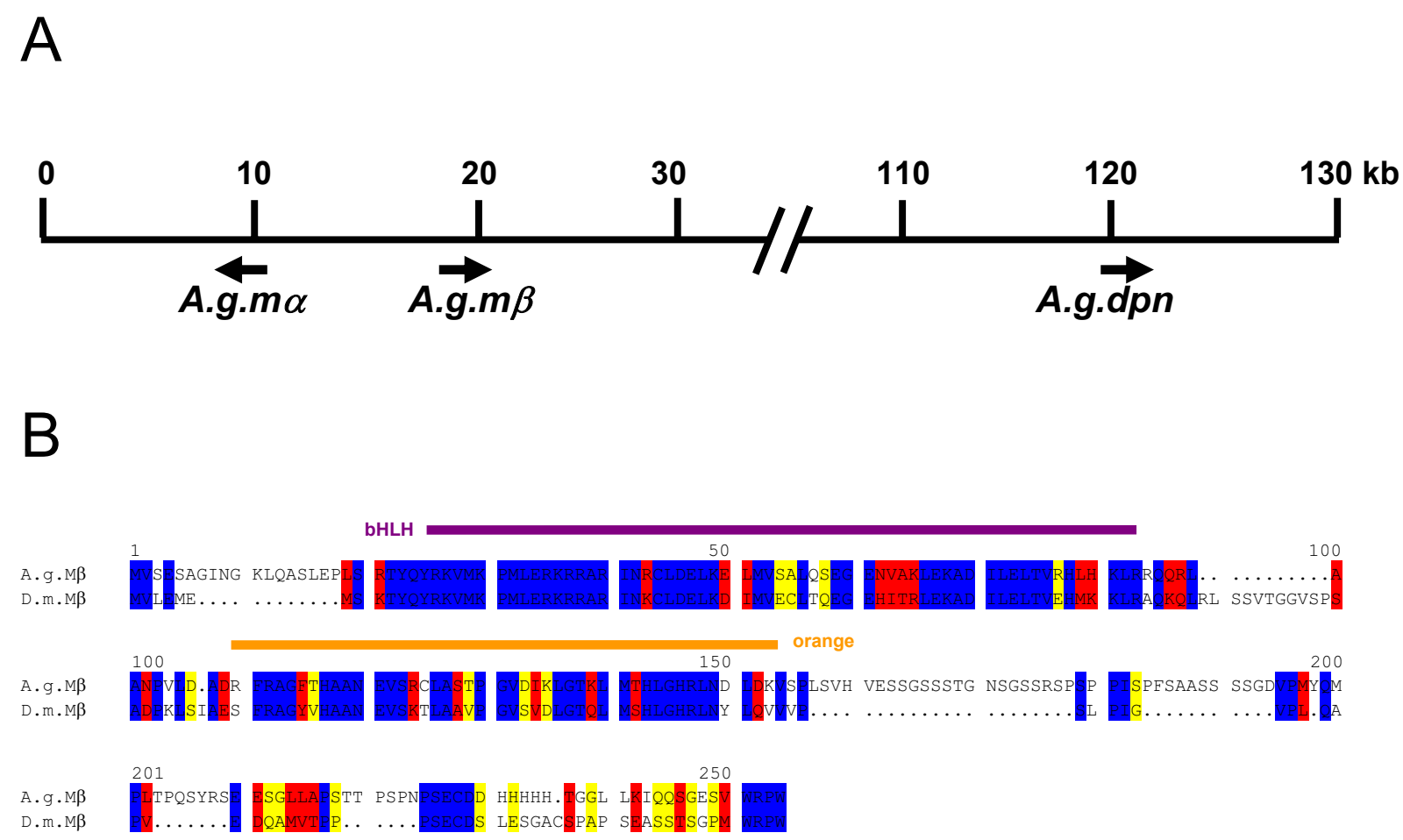

Figure 2

The $E(s p l)-C$ in mosquito A. gambiae. (A) The $E(s p l)-C$ in the mosquito is composed of two putative genes, $A . g . m \beta$ and A.g.m $\alpha$. Approximately $100 \mathrm{~kb}$ away a second bHLH coding gene was detected, however, the analysis predicted a close relationship to Deadpan and Hairy. Since a good fitting hairy ortholog is elsewhere in the mosquito genome, the gene is most likely a dpn ortholog. B) Alignment between A.g.M $\beta$ and D.m.M $\beta$ shows good conservation within the bHLH and the orange domains (marked) as well as the WRPW motif at the C-terminus. Identical residues are marked in blue; red shows highly related and yellow similar residues.

into the intron that maintains an open reading frame and ends with a WRPW motif. In this case, the E(spl) homolog of mosquito would be without intron and shares highest similarity to the $\mathrm{M} \beta / \mathrm{M} \gamma$ pair of $D$. melanogaster $(80.3 /$ $75.7 \%$ similarity and $69.6 / 67.4 \%$ identity, respectively). Based on the similarity, we propose that it corresponds to D.m. $m \beta$ (Fig. $2 \mathrm{~B}$ ) and named it therefore A.g. $m \beta$. Moreover, there is a single Brd-like gene in close proximity ( $\sim 8$ $\mathrm{kb}$ ) of A.g.m $\beta$ (Fig. 2A). This gene encodes a protein most similar to the $D$. melanogaster $M \alpha$ protein with almost $60 \%$ identity (Fig. 3A); therefore we named it A.g.m $\alpha$. It shares all features of the Brd-family proteins described earlier [14], including the amphipathic $\alpha$-helix domain in the N-terminal part (Fig. 3B).

Approximately $100 \mathrm{~kb}$ from the $3^{\prime}$ end of A.g.m $\beta$, we detected another sequence that might encode an $\mathrm{E}(\mathrm{spl})$ bHLH type protein (Fig. 2A; ENSANGG00000017548). However, the presumptive gene product is more highly related to D. melanogaster Deadpan (Dpn; 57\% identity) than to $\mathrm{E}$ (spl) bHLH M $\beta$ (52\% identity). The conservation extends beyond the amino acid sequence: we find the same intron/exon structure in this Anopheles gene as in the deadpan and hairy genes from Drosophila (see also Fig. 8a). We believe that this Anopheles protein corresponds to Dpn (A.g.Dpn) rather than to Hairy, since it shares little more than $47 \%$ identity with $D$. melanogaster Hairy. The best hit with the D. melanogaster Hairy protein is found on the second chromosome in Anopheles (A.g.h; $72 \%$ identity at protein level). Other genes of the Drosophila $E(s p l)-C$ were not detected nearby: maybe they are not conserved enough to be discovered like e.g. $m 6$ or they are located at totally different positions in the genome like gro.

\section{The $\mathbf{E}(\mathrm{spl})-\mathbf{C}$ in Apis mellifera}

Like in the mosquito, there is no extended $E(s p l)-C$ in the honeybee. In fact, we find a similar structure of one $\mathrm{E}(\mathrm{spl})$ bHLH type and one Brd-type gene that share highest 
Table I: Identity matrix of E(spl) bHLH proteins in D. melanogaster (\% similarity/identity)

\begin{tabular}{|c|c|c|c|c|c|c|c|}
\hline & $M \beta$ & $M \delta$ & M8 & M7 & M5 & M3 & $\varnothing$ \\
\hline$M \gamma$ & $75 / 68$ & $68 / 59$ & $69 / 57$ & $75 / 67$ & $72 / 61$ & $74 / 64$ & $72 / 63$ \\
\hline$M \beta$ & & $67 / 56$ & $70 / 61$ & $75 / 68$ & $71 / 60$ & $80 / 71$ & $73 / 64$ \\
\hline$M \delta$ & & & $64 / 50$ & $67 / 59$ & $65 / 50$ & $67 / 53$ & $66 / 55$ \\
\hline M8 & & & & $67 / 59$ & $80 / 73$ & $63 / 50$ & $69 / 58$ \\
\hline M7 & & & & & $67 / 61$ & $70 / 61$ & $70 / 63$ \\
\hline M5 & & & & & & $66 / 57$ & $70 / 60$ \\
\hline M3 & & & & & & & $70 / 59$ \\
\hline
\end{tabular}

$\varnothing=$ average similarity/identity (\%) of one bHLH member if compared to all others

homology to $M \beta$ and to $M \alpha$, respectively (Fig. 4A). The relative transcription orientation (head to head) is the same, suggesting that $m \beta$ and $m \alpha$ represent the ur-complex (compare Figs. 1A, 2A, 4A). However, the situation in honeybee is more complicated in several respects.

For example, the A.m.m $\alpha$ homolog is predicted to consist of five exons that code for a protein with 402 residues. This is considerably larger than the 138 residues of D.m.M $\alpha$. Again, we propose a different gene structure based on the analysis of the genomic DNA, where the translation extends into the first postulated intron and ends shortly afterwards. The encoded protein than consists of only 168 amino acids and terminates with residues that are very similar to the M $\alpha$ Drosophila homolog (M Q V A) (Fig. 3A). Moreover, it shows the typical amphipathic $\alpha$-helix domain in the N-terminal part (Fig. 3B). The other motifs are also conserved, the extreme C-terminus is, however, only similar (Fig. 3A). This protein shows also high similarity to Drosophila Twin of $\mathrm{m} 4$ (Tom) that might slightly exceed that to D.m.M $\alpha$ depending on the parameters used. As in mosquito, no other Brd-like protein was found in the honeybee database such that a Brd-complex seems non-existent, unlike in Drosophilids [33].

The Apis database annotates a single intron for the A.m. $m \beta$ gene that conforms to the GT AG rule. This is in contrast to all the Drosophila E(spl) bHLH genes that are intronless. Moreover, there are several possible start sites and it remains unclear which one is used. In D. melanogaster, there are other bHLH-WRPW encoding genes that contain introns, like deadpan ( $d p n)$ or Side. However, the respective protein sequences do not align well with A.m.M $\beta$, and the Apis genome contains predicted orthologs to these genes at other locations (see below). The A.m.M $\beta$ protein is also highly similar to melanogaster $\mathrm{M} \gamma$ with 72.3 versus $77.7 \%$ similarity and 65 versus $67.5 \%$ identity compared to D.m.M $\beta$ (Fig. 4B). These differences are extremely small. In fact, the Apis database proposes this gene as $m \gamma$ based on the similarity within the bHLH domain. However, we used for our comparison the entire protein sequences and calculated identity overall. Moreover, based on the E(spl)-C structure in Anopheles, we favour the hypothesis that $m \beta$ is the ancestral gene.

Within the $250 \mathrm{~kb}$ contig (GroupUn. 159), there are two further stretches that might encode $\mathrm{E}(\mathrm{spl})$ type bHLH proteins. One is located about $150 \mathrm{~kb}$ apart. This gene contains no introns and the encoded protein shows homology to both $\mathrm{M} \beta$ and $\mathrm{M} \gamma$. In this case, the similarity seems slightly higher to $M \gamma$ than to $M \beta$ (72.2 vs. $69.3 \%$ similarity and 66 vs. $62.7 \%$ identity). Therefore, we call the gene A.m.m $\gamma$ (Fig. 4A,B). Both FlyBase and BlastN give a higher score to $D$. melanogaster $\mathrm{M} \beta$ than to $\mathrm{M} \gamma$. Presumably, both use similar paradigms based on an alignment of only the best conserved sequences, whereas we used less stringent parameters (see Methods) to allow an alignment of the complete sequences. As to be expected, the two $\mathrm{E}(\mathrm{spl})$ honeybee proteins A.m.M $\beta$ and A.m.M $\gamma$ are highly related to each other with a similarity of $73 \%$ and an identity of $67 \%$. Interestingly, this numbers are very similar to those from a likewise comparison of the D. melanogaster $\mathrm{M} \beta$ and $\mathrm{M} \gamma$ homologs (Table 1).

Another $50 \mathrm{~kb}$ further up at position 50 (Fig. 4A), we found a short alignment to the $\mathrm{E}(\mathrm{spl}) \mathrm{bHLH}$ domain. However, there was no predicted gene, nor a start codon, nor a WRPW motif. Nearby at position $\sim 46 \mathrm{~kb}$ there is an open reading frame of 133 residues split by one intron that belongs to a predicted database gene consisting of five exons (GENSCAN00000025907; black in Fig. 4C). However, lacking any similarity to known genes of Apis or other species, this gene remained without functional prediction in the Apis database. In agreement, our searches in the FlyBase did not detect any similar sequences. However, we predict an E(spl) bHLH-type protein encoded by this gene region: extension of the open reading frame into the adjacent intron ends in WRPW (Fig. 4C, blue exons). The bHLH encoding sequences (purple in Fig. 4C) are located within the second predicted intron of the putative gene shown in black. There are respective exon/intron boundary consensus sequences to allow for a single transcript that contains the bHLH domain, an orange domain and ends with the WRPW motif (Fig. 4C). This third E(spl) 


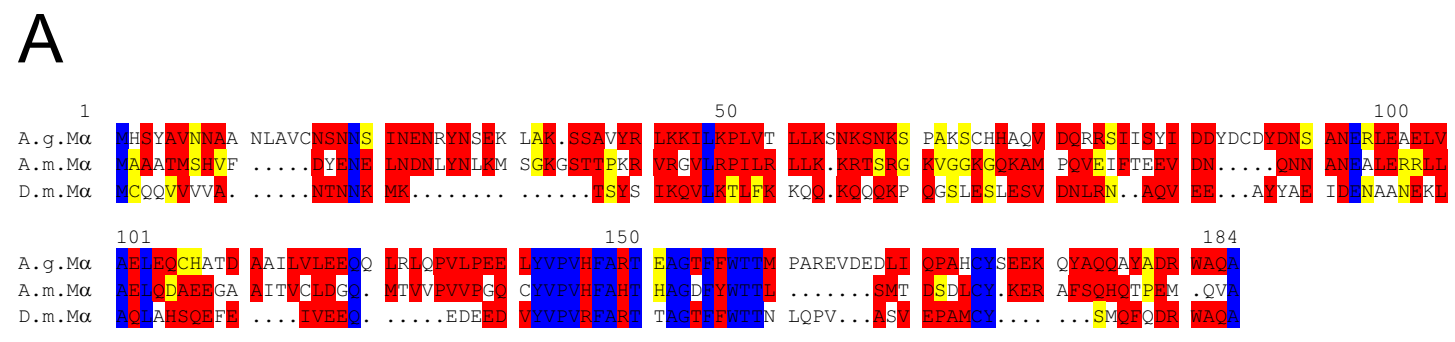

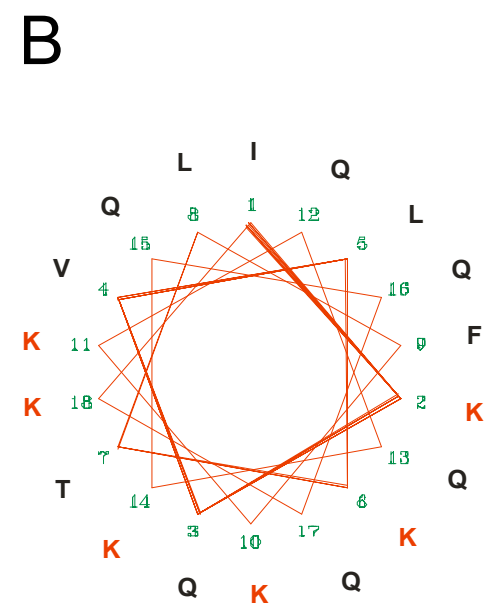

D. melanogaster

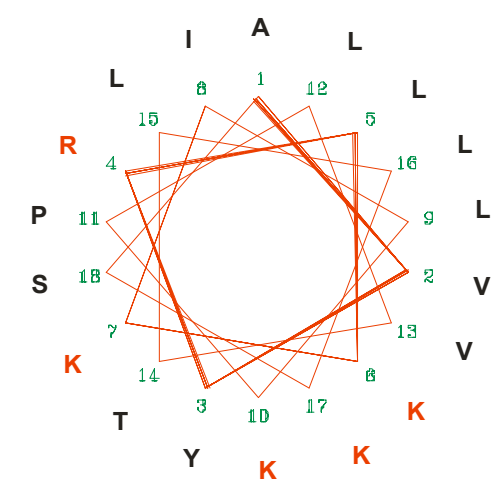

A. gambiae

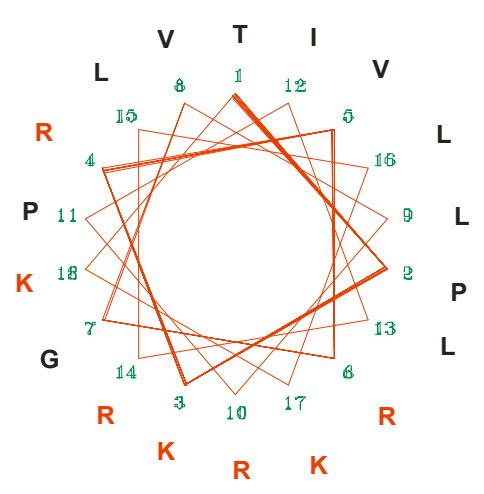

A. mellifera

\section{Figure 3}

Conservation of the Brd-family member M $\alpha$. A) Alignment of the presumptive M $\alpha$ proteins of $D$. melanogaster, A. mellifera and A. gambiae. Although the alignment reveals not much identity (blue), the postulated features that typify Brd-proteins are present [ I4]. Red, highly related and yellow, similar residues. B) M $\alpha$ contains a typcial amphipathic $\alpha$-helix with high concentration of lysine $(K)$ and arginine $(\mathrm{R})$ residues on one side of the wheel (red).

bHLH gene would comprise five exons. In fact, in the update May 2005 of the Ensembl honeybee database, the Ensembl automatic analysis pipeline predicts a very similar protein, however, with a different $\mathrm{N}$-terminus and slightly smaller third and fourth exons. This gene than consists of four exons which would be similar to D. melanogaster hairy and $d p n$ that contain two introns. However, the encoded protein is most similar to the $\mathrm{E}(\mathrm{spl})$ protein $\mathrm{M} \beta$, so we call the gene A.m. $m \beta$ '. Since Apis hairy and $d p n$ are found elsewhere in the genome (see below), we propose that the E(spl)-C in Apis mellifera consists of the urcomplex plus two further $E(s p l)$ bHLH genes most closely related to $m \gamma$ and $m \beta$. No other genes of the Drosophila $E(s p l)-C$ are present in that of the honeybee. We find a highly conserved Groucho ortholog, however, at a completely different position in the genome.

\section{Conservation of other Hairy/E(spl)-like proteins known from Drosophila}

In total, 12 genes are known in D. melanogaster to encode Hairy/E(spl)-like proteins, i.e. bHLH proteins that also have the orange domain and a WRPW-type Gro-binding motif (see Table 2). Apart from the seven E(spl) bHLH proteins, these include Hairy, Deadpan, Side, Hey and 
A

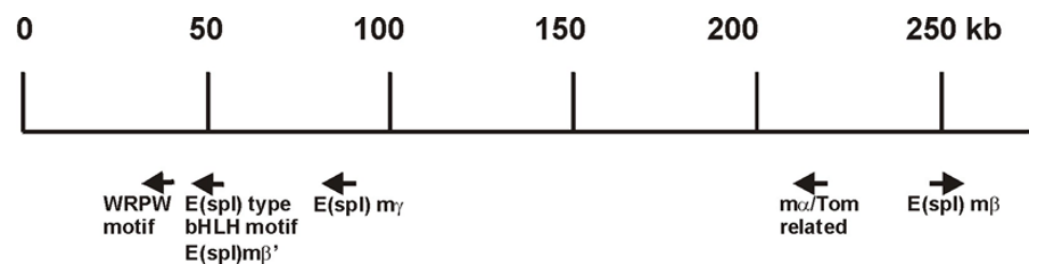

B
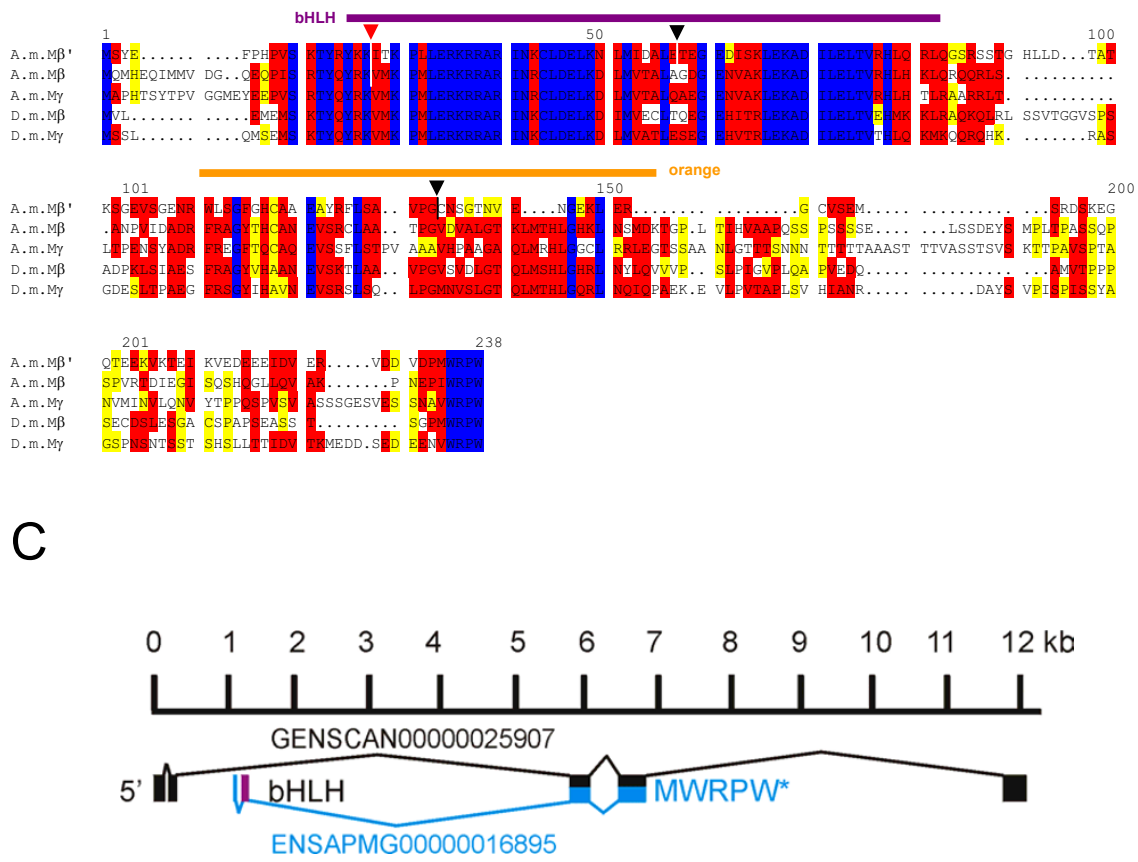

\section{Figure 4}

The E(spl)-C in Apis mellifera. A) A genomic region spanning about $250 \mathrm{~kb}$ (GroupUn. I59; numbers are correspondingly) contains 3 presumptive $E(s p l) b H L H$ genes and one $m \alpha$ related gene. The gene at position $\sim 250 \mathrm{~kb}$ encodes a bHLH protein with best overall identity to D.m.MP. This gene is disrupted by an intron inside the bHLH domain (dash and red arrowhead in B). Close by, at position $220 \mathrm{~kb}$ a $m \alpha$ related coding region is found. Based on high similarity to D.m.M $\alpha$ and its close neighbourhood to A.m.m $\beta$, we named it A.m.m $\alpha$. A second intronless $E(s p l)$ like bHLH coding gene is located at position $\sim 90 \mathrm{~kb}$. We name it A.m.m $\gamma$ since the encoded protein shows best overall identity to the D.m.M $\gamma$. Approximately $50 \mathrm{~kb}$ away we detected sequences encoding an $\mathrm{E}(\mathrm{spl})$ like bHLH domain (A.m.MP') and a long open reading frame ending with a WRPW motif. The Ensembl honeybee database annotated a gene with five introns spanning $12 \mathrm{~kb}$ within this region but missed the respective motifs (GENSCAN00000025907). We propose a different gene structure; see Figure $C$ for details. B) Alignment of the three putative honeybee bHLH proteins (A.m.M $\beta^{\prime}$, A.m.M $\beta$, A.m.M $\gamma$ ) with D. melanogaster $M \beta$ and $M \gamma$ proteins is shown. Identical residues are marked in blue; red shows highly related and yellow similar residues. Intron positions are marked with a triangle above and a dash in the respective A. mellifera sequence. C) Structure of the $12 \mathrm{~kb}$ GENSCAN00000025907 region. Black shows the Ensembl gene annotation, and blue the new ENSAPMG000000I6895 annotation. Purple highlights a second exon that encodes part of the bHLH domain, the open reading frame extends into the predicted intron of GENSCAN00000025907 and terminates with WRPW. 
Her [34]. Moreover, there is similarity to Stich1/Sticky which has a bHLH and an orange domain but not the typical Gro-binding motif [35]. Since the number of $E(s p l)$ bHLH genes is not conserved in honeybee and mosquito, it was interesting to ask whether all the other genes were present. We searched the Ensembl database with the respective $D$. melanogaster protein sequences and found orthologs of all genes except of $\mathrm{Her}$ in both species (see Table 2). However, most of the predictions are incomplete. We know from $D$. melanogaster that these genes contain introns, which complicates the search for potential coding sequences within genomic DNA. Thus, our protein sequence predictions are uncertain. With the sole exception of Dpn, all the proteins are better conserved between Drosophila and Anopheles than between Drosophila and Apis, confirming the evolutionary relationship. The best conserved proteins are Hey and Hairy. The Hey orthologs are $76 \%$ identical between Drosophila and Anopheles and 66\% between Drosophila and Apis and the Hairy orthologs between $72 \%$ and $65 \%$, respectively. Less conservation is found for Side, Dpn and Stich1 (62/57 Side, 57/59 Dpn and $60 / 57$ Stich $1 ; \%$ identity comparing fly with mosquito and honeybee, respectively). All proteins share the bHLH and orange domains. The WRPW motif of Hairy, Dpn and Side as well as the YRPW motif of Hey is present in the orthologs.

\section{The Achaete-Scute complex in Drosophilids}

The Achaete-Scute complex (AS-C) is well conserved in D. virilis: all four genes, achaete (ac), lethal of scute (l'sc), scute $(s c)$ and asense (ase) are found in the same order and orientation on the X-chromosome (Fig. 5A). Like in D. melanogaster, the genes are without introns. All proteins share the typical bHLH motif of the AS-C proteins and this domain reveals the lowest evolutionary rate. However, compared with the bHLH proteins of the E(spl)-C the bHLH proteins of the AS-C evolve faster. The complex can be separated into two clusters that are distinguished by their rate of conservation. On one hand, L'sc and Sc are well conserved with an identity between $D$. melanogaster and D. virilis of more than $75 \%$ and on the other hand Ac and Ase with an identity of less than $69 \%$ (Fig. 5A). Note that the highest divergence that was found between these two species in the $E(s p l)-C$ was for M8 with still almost $81 \%$ identity.

Of the four $A S-C$ gene members in $D$. melanogaster, ase stands out because it is much larger than the other three. In D. virilis, the size increase is even more striking: D.v.Ase is predicted to comprise 619 residues, whereas D.m.Ase is only 486 residues in length (Fig. 5B). This extension of more than $20 \%$ additional residues is caused by multiple insertions of repetitive sequences that code for polyglutamine $(\mathrm{Q})$, poly-alanine $(\mathrm{A})$ and poly-asparagine $(\mathrm{N})$ stretches (Fig. 5B). Like in D. melanogaster the unrelated gene pepsinogen-like ( $p c l$ ) is located between l'sc and ase (Fig. 5A).

\section{The AS-C in Anopheles gambiae}

In the mosquito, we find only two potential achaete-scute like genes that are in close neighbourhood of less than 30 $\mathrm{kb}$. Interestingly, like in Drosophila they are located on the $\mathrm{X}$-chromosome. One of them encodes a protein that is very similar to L'sc and Sc proteins not only within the bHLH domain but also at the C-terminus (Fig. 6A). This is not unexpected because the C-terminus is involved in transcriptional activation as well binding of $\mathrm{E}(\mathrm{spl}) \mathrm{bHLH}$ proteins [36]. The BestFit program gives a higher score to L'sc (64\% identity) than to Sc (57\% identity). However, closer inspection reveals that some protein regions are more similar to D.m.Sc and others more to D.m.L'sc (Fig. $6 \mathrm{~A})$ suggesting common ancestry for this gene pair. The Anopheles data base predicts an intron, which however retains the open reading frame. In fact, Wülbeck and Simpson [37] cloned and sequenced the respective A.g.ash cDNA and showed that it is intronless. We detected three conservative amino acid exchanges between the published A.g.Ash protein sequence and that obtained from translating the database genomic DNA, namely at position 8 (M-L), position 189 (T-S) and position $311(\mathrm{Q}-\mathrm{H})$.

The second presumptive gene has two predicted introns. The derived amino acid sequence shares between 54\% and $57 \%$ identity with all four D. melanogaster AS-C proteins. By searching the $D$. melanogaster genome with the predicted protein sequence, the best hit was found to Ase protein (Fig. 6B). Accordingly, the Anopheles database defined it as ase homolog and so we named it A.g.ase. We note, however, that a precise appointment is difficult based on the lack of a significant similarity at the C-terminus which normally allows the distinction between the $A S-C$ members.

\section{The AS-C in honeybee}

Like in the mosquito, the honeybee genome encodes only two AS-C like proteins. The two transcription units are the predicted Ensembl genes ENSAPMG00000003261 and ENSAPMG00000003265 (Table 2). They are located in the scaffold group 10.3 and are approximately $40 \mathrm{~kb}$ apart from each other. The former encodes a protein that is highly similar to the L'sc/Sc protein pair, so we named it A.m.ash (Fig. 7A). In contrast to the Drosophila AS-C genes, A.m.ash is predicted to contain a single intron which however, retains an open reading frame. Therefore, like in mosquito the encoded protein could be significantly larger. We thus aligned the protein sequences of A.m.Ash with and without translation of the predicted intron with D.m.L'sc (Fig. 7A). Since the open reading frame of the presumptive intron is translated primarily into serine residues, no alignment with the D.m.L'sc protein was possi- 
ble for this part, supporting the intron prediction. We note that there are two more exon/intron boundary consensus sequences within the predicted intron (arrows in Fig. 7A). If these were used instead of the ones predicted, the intron would be somewhat smaller and A.m.Ash accordingly 25 amino acids larger. As shown in Fig. 7A, the resultant protein would be more similar in size to melanogaster L'sc protein and moreover, share additional similarities in this part of the protein. The Apis database does not provide a start for A.m.Ash, which we could deduce however from the alignment with the D. melanogaster protein.

Interpretation of the second gene is much more difficult. The annotation by Ensembl automatic pipeline using GeneWise model based on either protein or aligned EST's resulted in a coding sequence within a single exon that lacked both start and stop codons, however, aligned well with AS-C bHLH domains. We thus extended our studies into the surrounding genomic DNA, where we detected one further open reading frame. The deduced amino acid sequence matched well with the N-terminal part of D. melanogaster Ac and Ase proteins. However, the C-terminus did not align convincingly (Fig. 7B/GW). Another gene prediction using Chris Burge's Genscan program [38] gave a transcript of 8 exons spanning over $8 \mathrm{~kb}$ of genomic DNA. The translation gave a larger protein again without start methionine that contained the single exon predicted by the GenWise model (Fig. 7B/GS). Again there was very little similarity in the C-terminal part of this and Drosophila AS-C proteins (Fig 7B). Because Genscan could not predict the protein start, we propose a combination of both models with the $\mathrm{N}$-terminus as shown in the A.m.Ase/GW sequence and the C-terminus as in A.m.Ase/ GS that might, however, end shorter than shown in Fig. 7B.

The high divergence from the Drosophila AS-C proteins renders precise predictions very difficult. In fact, under standard conditions like FlyBase BlastN only parts of the bHLH domain can be identified. Comparison with the $D$. melanogaster AS-C proteins gave minimally different scores, with the highest score found with D.m.Ac followed by D.m.Ase, dependent on the parameters. For example, the Apis database finds best scores with melanogaster Sc and L'sc. We named this gene A.m.ase by its similarity notably in the N-terminal part and based on the arrangement of the mosquito AS-C.

\section{Conservation of predicted regulatory elements in E(spI)-C and AS-C}

Neurogenesis in Drosophila is subjected to various levels of regulation. As described in the introduction, $\mathrm{E}(\mathrm{spl}) \mathrm{bHLH}$ proteins repress proneural gene activity. Negative regulation is brought about by repression of transcription as well as at the protein level [36,39-42]. A further mode of regulation involves RNA:RNA duplexes [43]. These are formed by small sequence stretches located in the $3^{\prime}$ untranslated region (3' UTR) of the mRNAs of proneural $A S-C$ genes and different members of the $E(s p l)-C$. For example, l'sc mRNA contains so-called proneural boxes (PB-box) (GGAAGAC) which bind to the GY-boxes (GUCUUCC) of $E(\mathrm{spl}) \mathrm{m} 4 \mathrm{RNA}$ [43]. We searched the genomic sequences adjacent to the coding sequences for respective regulatory elements and found them in D. virilis: there are two PB boxes in D.v.l'sc and GY-boxes in the 3'UTR of the predicted virilis $E(s p l)$ genes $m 3, m 4, m 5$ and $m \gamma$ just as in D. melanogaster and meanwhile published by Lai et al. [33]. Moreover, it has been shown that E(spl) genes are direct targets of the Notch signal involving the DNA binding protein $\mathrm{Su}(\mathrm{H})[11,13,15,19]$. Accordingly, there are potential $\mathrm{Su}(\mathrm{H})$ binding sites (C/TGTGA/GGA) in all D. melanogaster $E(s p l)$ genes including $m 2$ and $m 6$ $[14,15]$, which are also present in the respective virilis orthologs [44]. However, the predicted binding sites for proneural bHLH activators (E box: GCAGGTG) [14] are less well conserved during evolution. Whereas the D. virilis $m 2$ and $m 4$ orthologs contain such a regulatory element, we found no sequence fitting the E-box consensus in either the D.v.m6 or D.v.ma sequence.

In mosquito and honeybee, regulatory elements of RNA:RNA duplex-type were not detected. None of the two $A S-C$ genes of either Apis or Anopheles contained PB-box like sequences in the $3^{\prime}$ UTR, albeit the highly diverged sequence and gene structure of A.g.ase does not allow to definitely exclude their presence. Since there is no predicted $m 4$ ortholog in Anopheles and Apis, we looked at the $3^{\prime}$ end of the $m \alpha$ gene as the single Brd-family member. However, in none of these gene sequences did we find GY type boxes like in Drosophila. We note that some of the predicted gene structures are still incomplete and a search for small sequence stretches is notoriously difficult if one allows for variations. Therefore, there is the formal possibility that we have missed these sites.

\section{Discussion}

\section{The Enhancer of split complex}

Extensive genome analyses in the recent years revealed that there are not many examples of large gene complexes that are widely conserved. Prominent examples are the HOX ( $\underline{\text { homeobox }}$ ) complexes, which contain homeotic genes in Drosophila. HOX complexes are well conserved in metazoans despite some variations in gene number. HOX-genes encode regulatory proteins with specific individual functions and mutations affect different aspects of the body plan [45]. Not surprisingly, it is almost only the homeodomain, which serves as sequence-specific DNA binding motif that is conserved amongst different species [46]. In contrast, similarity amongst bHLH proteins 
Table 2: Gene annotation used by the respective databases

\begin{tabular}{|c|c|c|c|c|}
\hline D. melanogaster & $\begin{array}{l}\text { D. pseudoobscura } \\
\text { (contig } 4374 \text { Contig 4847) }\end{array}$ & D. virilis & Apis mellifera & Anopheles gambiae \\
\hline D.m. $m \delta($ CG8328) & D.p. $m \delta(178$ 652-178 089) & D.v. $m \delta$ & - & - \\
\hline D.m. $m \gamma(\mathrm{CG} 8333)$ & D.p. $m \gamma(175$ 594-I74 953) & D.v. $m \gamma$ & A.m. $m \gamma($ ENSAPMG0000004887) & - \\
\hline D.m. $m \beta$ (CGI4548) & D.p. $m \beta(169335-169934)$ & D.v. $m \beta$ & A.m. $m \beta$ (ENSAPMG000000488I) & A.g. $m \beta$ (ENSANGG000000I760I) \\
\hline D.m. $m \alpha$ (CG8337) & D.p. $m \alpha(163786-163358)$ & D.v. $m \alpha$ & A.m. $m \alpha$ (GENSCAN000000I764) & A.g. $m \alpha$ (SNA000000II40I) \\
\hline D.m. $m$ I (CG8342) & D.p. $m$ I (I58 207-I58 734) & D.v. $\mathrm{ml}$ & - & - \\
\hline D.m. m2 (CG6104) & D.p. $m 2$ (I57 0I2-157 407) & D.v. $m 2$ & - & - \\
\hline D.m. m3 (CG8346) & D.p. $m 3(15|792-15|$ |36) & D.v. $m 3$ & - & - \\
\hline D.m. m4 (CG6099) & D.p. m4 (149 049-149 516) & D.v. $m 4$ & - & - \\
\hline D.m. m5 (CG6096) & D.p. m5 (|42 32I-|42 88I) & D.v. $m 5$ & - & - \\
\hline D.m. m6 (CG8354) & D.p. $m 6(137883-137656)$ & D.v. $m 6$ & - & - \\
\hline D.m. $m 7$ (CG836I) & D.p. m7 (|34 008-|33 39|) & D.v. $m 7$ & - & - \\
\hline D.m. m8 (CG8365) & D.p. $m 8$ (130 I85-129 628) & D.v. $m 8$ & - & - \\
\hline D.m. gro (CG8384) & D.p.gro (I 48 473-I I 7 489) & D.v. gro & A.m. $m \beta$ (ENSAPMG000000I6895) & - \\
\hline D.m. stich I (CGI7I00) & not analysed & not analysed & $\begin{array}{l}\text { A.m. stich I } \\
\text { (ENSAPMG00000005857) }\end{array}$ & A.g. stich I (ENSANGG000000I6365) \\
\hline D.m. side (CGI0446) & not analysed & not analysed & A.m. side (ENSAPMG0000000088) & A.g. side (ENSANGG000000I4329) \\
\hline D.m. dpn (CG8704) & not analysed & not analysed & A.m. dpn (ENSAPMG0000000455I) & A.g. dpn (ENSANGG000000I7548) \\
\hline D.m. Hey (CGIII94) & not analysed & not analysed & A.m. Hey (ENSAPMG0000000726) & A.g. Hey (ENSANGG0000002 I744) \\
\hline D.m. Her (CG5927) & not analysed & not analysed & - & - \\
\hline D.m. $h(C G 6494)$ & not analysed & not analysed & A.m. $h$ (ENSAPMG00000004545) & A.g. $h$ (ENSANGG000000।8369) \\
\hline D.m. ac (CG3796) & not analysed & D.v. $a c$ & - & - \\
\hline D.m. sc (CG3827) & not analysed & D.v. sc & - & - \\
\hline D.m. I'sc (CG3839) & not analysed & D.v. I'sc & A.m. ash (ENSAPMG0000000326I) & $\begin{array}{l}\text { A.g. ash } \\
\text { (ENSANGG00000010650(Q95VY6) }\end{array}$ \\
\hline D.m. ase (CG3258) & not analysed & D.v. ase & A.m. ase (ENSAPMG00000003265) & A.g. ase (ENSANGG000000I 534I) \\
\hline D.m. da (CG5I02) & not analysed & not analysed & A.m. da (ENSAPMP00000005673) & $\begin{array}{l}\text { A.g. da (ENSANGEST0000036169l/ } \\
\text { SNAP0000000 I 2539) }\end{array}$ \\
\hline D.m. Ocho (CG5।38) & not analysed & not analysed & - & - \\
\hline D.m. Tom (CG5 I85) & not analysed & not analysed & - & - \\
\hline D.m. Brd (CG3096) & not analysed & not analysed & - & - \\
\hline
\end{tabular}

(-, not found)

encoded by the $E(s p l)-C$ extends over the entire length, even within the same species indicating rather recent duplication events. The $D$. melanogaster proteins M8/M5 and $\mathrm{M} \beta / \mathrm{M} 3$ are most similar with over $70 \%$ identity, whereas $M \delta$ is the most diverged. However, $M \delta$ still shares at least $50 \%$ identity with other $E(s p l)$ bHLH protein members (see pair wise comparison in the identity matrix of $\mathrm{M} \delta$ with M8, M5 and M3; Table 1). More interesting is the analysis of the overall similarity amongst these proteins. Here, any one of the proteins is compared with the other six and the result is averaged. Clearly, $M \beta(73 / 64 \%$, similarity/identity) closely followed by $M \gamma(72 / 63 \%)$ is most similar to all others, whereas $M \delta(66 / 55 \%)$ shows the lowest values (Table 1). One interpretation might be that the different bHLH genes evolved by duplication out of $m \beta$ or $m \gamma$. Remarkably, these two bHLH proteins besides M3 are the best conserved in the three Drosophila species (Fig. 1A). We would like to postulate that these are the most ancient proteins with the most general function and, therefore, the highest selection pressure. This hypothesis is supported by the finding that $m \beta$ has the most general expression pattern from which the others can be derived by a decrease of gene activity [19]. The conspicuous conservation of M3 might hint to an important function during egg development as this gene is expressed also maternally $[17,22]$. The high degree of conservation of all $E(s p l)$ bHLH orthologous proteins in Drosophilids, which is clearly higher than the similarity within this protein family in D. melanogaster, indicates specific and nonredundant roles during development (see also [23]). Some of these functions have been identified in the past $[19,39]$. It is conceivable that regulatory sequences were not duplicated or evolved more rapidly so that we now find highly dynamic expression patterns of these genes.

\section{The ancestral E(spl)-C is composed of $\mathrm{m} \beta$ and $\mathrm{m} \alpha$}

As outlined above, $m \beta$ appears to be the ancestral bHLH gene of the $E(s p l)-C$ in Drosophilids based on its great 


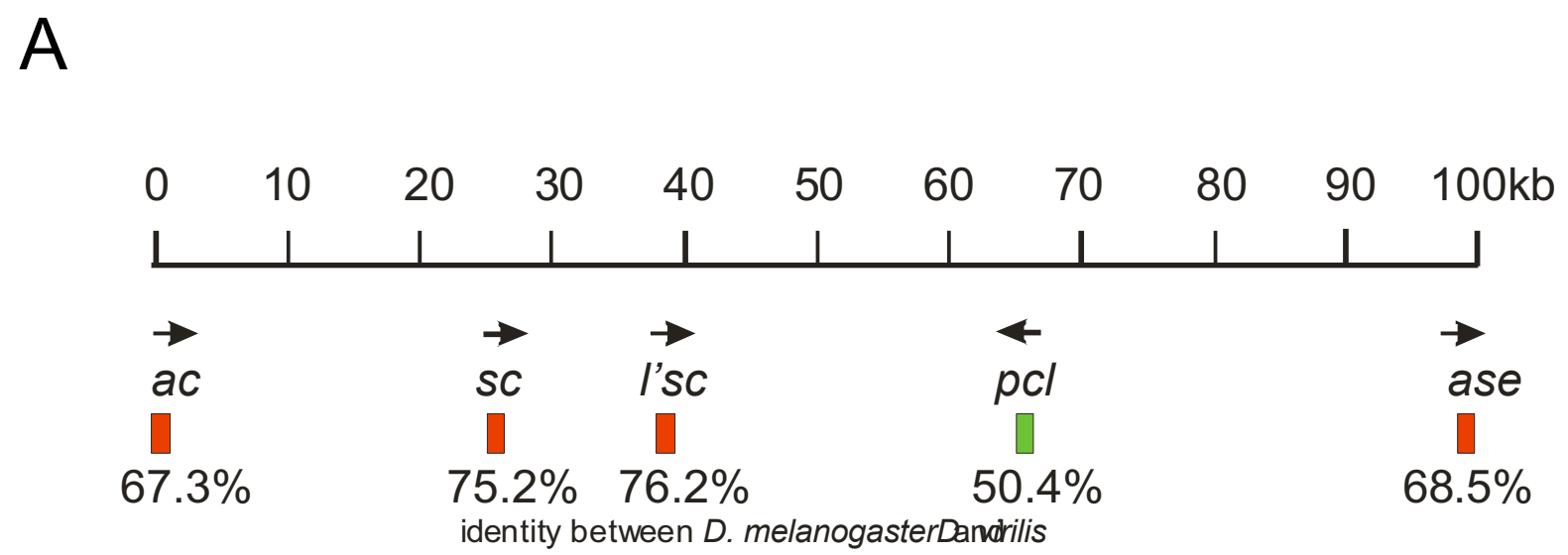

B

D.m.Ase D.v.Ase
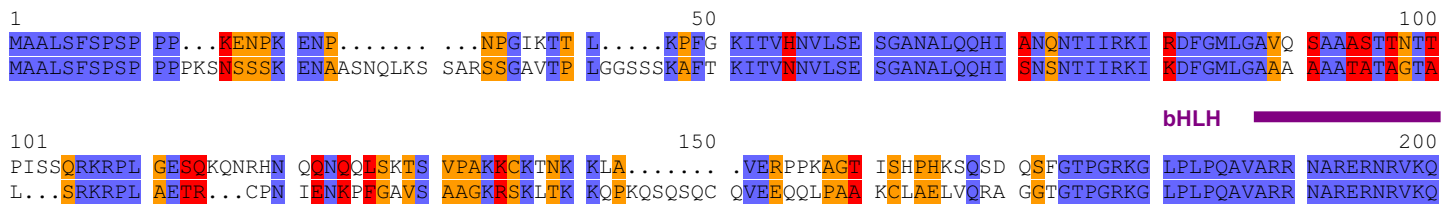

D.m.Ase
D.v.Ase

$101 \quad 150$

... VERPPKAGT ISHPHKSQSD QSEGTPGRKG

$\begin{array}{ll}\text { LPLPQAVARR } & \text { NARERNRVKQ } \\ \text { LPLPQAVARR } & \text { NARERNRVKQ }\end{array}$

D.m.Ase 201
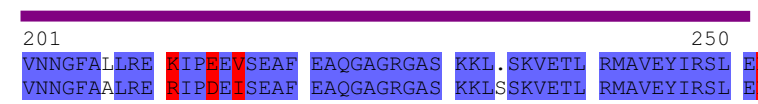

EKLLGFDFPP
ENLLG
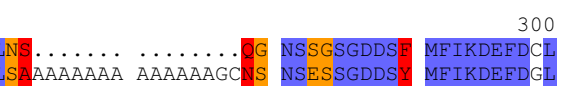

D.m.Ase
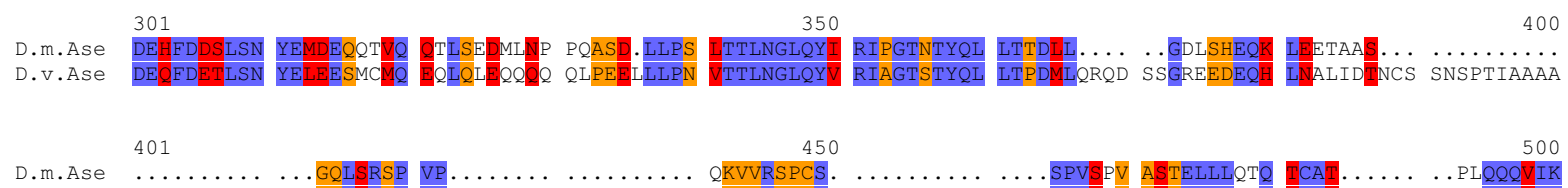

401

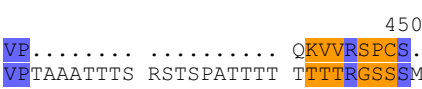

450

...SPVSPV ASTELLLQTQ TCAT....... . . PLQQQVIK
VAVSPVAVA VANELLL. . Q ACAAQQQQQQ
QQQQQQQLIK

D.m.Ase

501

D.m.Ase 60

D.v.Ase

601
DESMIDAIDW WEAHAPKSNG ACTNLS.V
DESMIDAIDW WEAHTPKSDA AAGAVAGI

\section{Figure 5}

The AS-C of D. virilis. A) The AS-C is highly conserved between D. melanogaster and D. virilis concerning gene number, transcript orientation and overall size. At the protein level (\% identity) the best conservation is found between the Sc and L'sc orthologs. However, the conservation rate is lower compared with the $\mathrm{E}(\mathrm{spl}) \mathrm{bHLH}$ proteins (see Fig. I). The pcl gene, although only $50.4 \%$ identical, is found between l'sc and ase. Because of gaps in the genomic virilis sequence, a scheme of the $D$. melanogaster complex is shown. B) Alignment of the Ase protein orthologs of D. melanogaster and $D$. virilis. Note the extension of the D.v.Ase protein by repetitive amino acid stretches composed of poly N, poly $Q$ and poly $A$. The best conservation is found within the bHLH domain (purple). Identical residues are marked in blue; red shows highly related and yellow similar residues. 


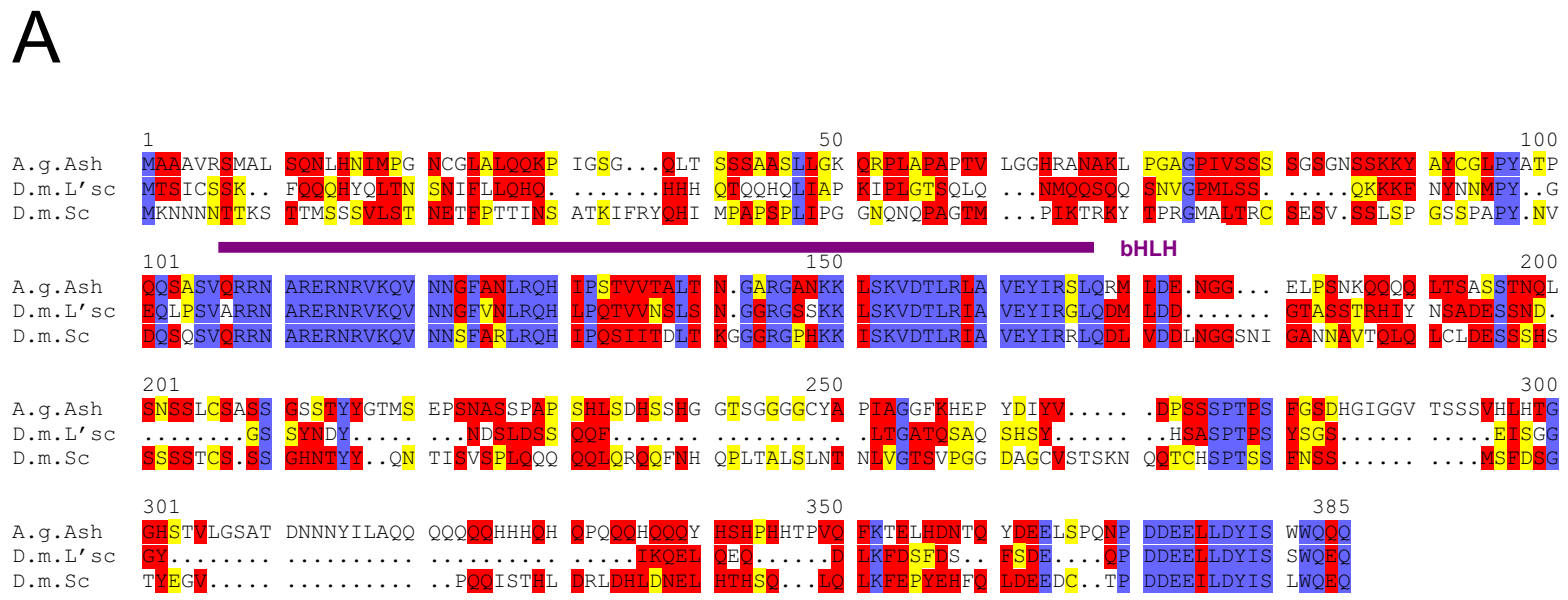

B

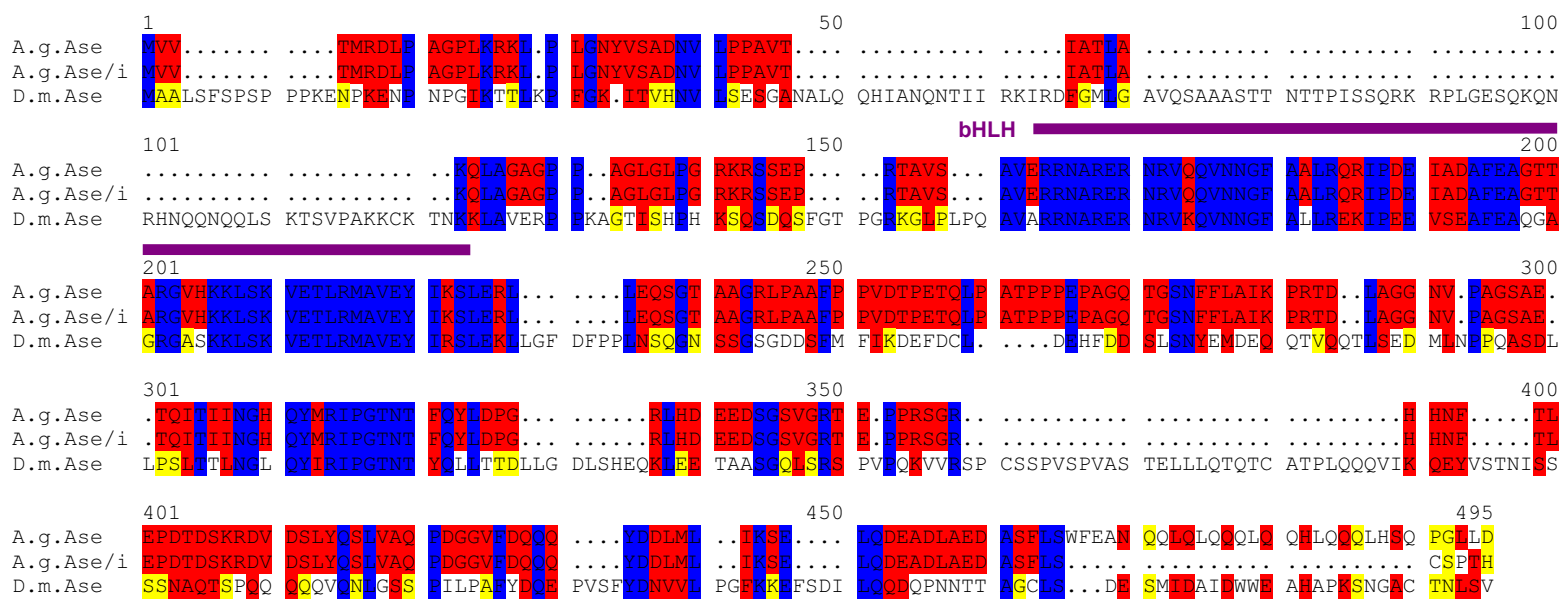

\section{Figure 6}

The AS-C in A. gambiae. A) Alignment of the achaete-scute homologous protein of A. gambiae (A.g.Ash; [36]) with melanogaster L'sc and Sc proteins. Note the high conservation of the bHLH domain (purple) and the very C-terminus. Comparison over the entire length gives a higher identity score to D.m.L'sc, however, the alignment shows also regions that are more similar to D.m.Sc. B) Alignment of the neighbouring bHLH gene product from A. gambiae with D. melanogaster Ase. The alignment is shown to A.g.Ase/i (database predicted version without intron) and A.g.Ase (second intron translated). The second predicted intron comprises almost $2.5 \mathrm{~kb}$ and ends with an exon translated into five residues (CSPTH; in A.g.Ase/i). Translation into this intron leads to A.g.Ase that is similar in size and in its terminus to D.m.Ase. Highest conservation is found in the bHLH domain (purple). Identical residues are marked in blue; red shows highly related and yellow similar residues. 


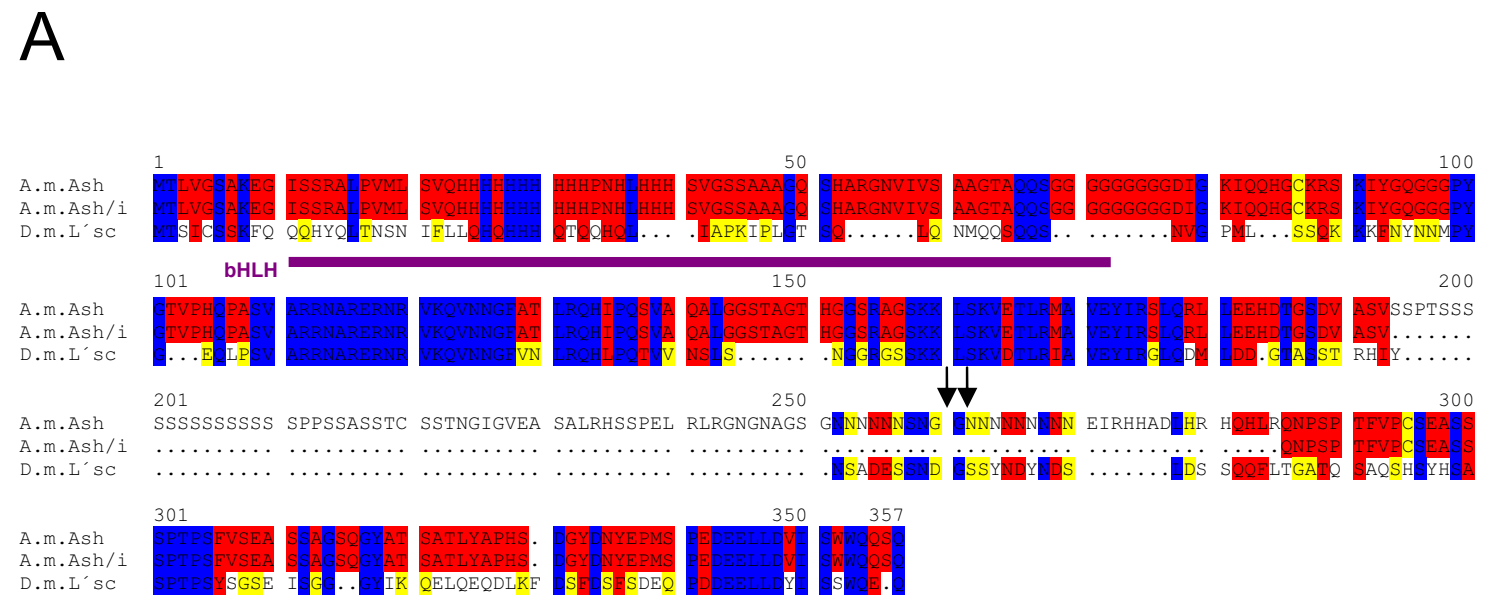

B

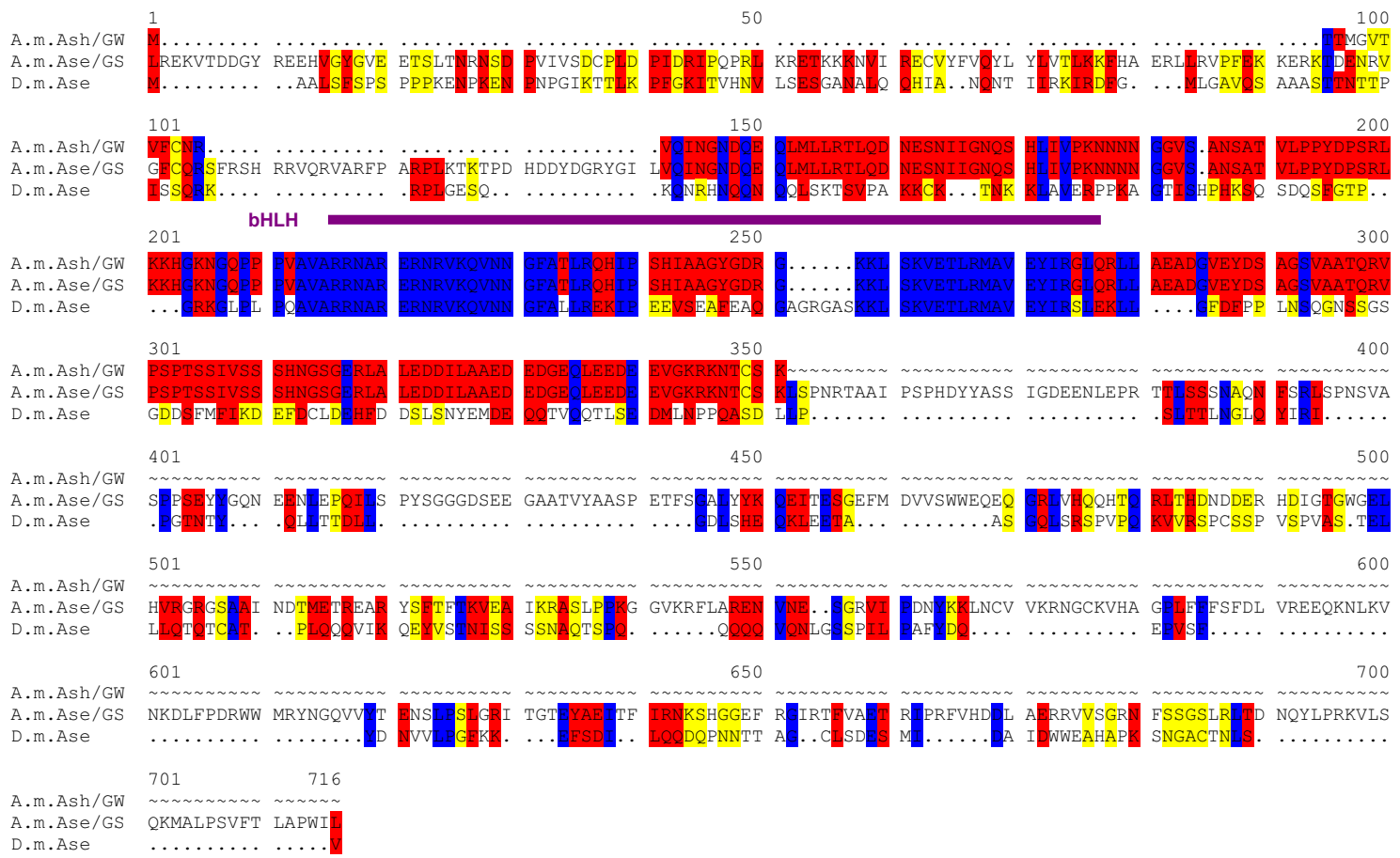

\section{Figure 7}

The AS-C in A. mellifera. A) Comparison of D.m.L'sc with the predicted A.m.Ash protein. Two forms were compared, without intron sequence, A.m.Ash/i, and with translated intron, A.m.Ash. Arrows mark additional splice consensus sites. B) Within $40 \mathrm{~kb}$ of A.m.ash, there is a second potential gene encoding a widely diverged bHLH protein. Two different programs were used for gene prediction that gives A.m.Ase/GS (Chris Burge's Genscan program) and A.m.Ase/GW (GeneWise model); both predicted proteins were aligned with D.m.Ase. Decent conservation is only found in the putative bHLH domains (purple). Identical residues are marked in blue; red shows highly related and yellow similar residues. 
similarity with all the other bHLH proteins. This assumption is strongly supported by the sequence conservation of the $\mathrm{E}(\mathrm{spl}) \mathrm{bHLH}$ proteins in A. gambiae and A. mellifera. The single $\mathrm{E}(\mathrm{spl}) \mathrm{bHLH}$ protein encoded by the mosquito genome has the highest identity to $\mathrm{M} \beta$. The genome of honeybee contains three prospective genes that encode proteins most highly related to $\mathrm{E}(\mathrm{spl}) \mathrm{D} . \mathrm{m} . \mathrm{M} \beta$ and D.m.M $\gamma$. All three are clustered within a single sequence contig, albeit they span a large segment of about $250 \mathrm{~kb}$, whereas the whole $E(s p l)-C$ in D. melanogaster comprises roughly $50 \mathrm{~kb}$. Despite the fact that two of these genes possess introns just within the bHLH domain and at positions close to the ones found in the D. melanogaster genes $d p n$, hairy or Her (Figs. 4, 8), the amino acid sequence similarity classifies them clearly as $\mathrm{E}(\mathrm{spl}) \mathrm{bHLH}$ proteins. A comparison of Anopheles and Apis proteins reveals, that the presumptive $M \beta$ homologs have highest similarity $(83 \%)$ and identity $(76 \%)$, whereas the protein that we classified as A.m.M $\gamma$ is just $70 \%$ similar and $66 \%$ identical to A.g.M $\beta$.

In Drosophilids, $m \alpha$ is located close to $m \beta$ and is transcribed in the opposite direction (head to head; Fig. 1A). This arrangement is likewise found in Anopheles and Apis (Figs. 2A, 4A). Notably, A. $m . m \alpha$ is next to A. $m . m \beta$, whereas the two Apis A.m.m $\gamma$ and A.m.m $\beta$ ' genes are much further apart (Fig. 4). We find this arrangement to be very ancient. In the beetle Tribolium, which on the tree of evolution is found even more deeply rooted ( $300 \mathrm{Myr}$ to Dipterans [32]), two similar genes coding for $\mathrm{M} \beta$-like proteins ( $\sim 65 \%$ and $\sim 67 \%$ identity to D.m.M $\beta$ ) are found and one is within $\sim 18 \mathrm{~kb}$ to a gene coding for an M $\alpha$-like protein ( $\sim 52 \%$ identity to D.m.M $\alpha$ ) (unpublished data derived from the Tribolium database). We postulate that the ur-complex consisted of these two ancestral genes, $m \alpha$ and $m \beta$. It is intriguing that they belong to the two different classes of Notch-responsive genes in the $E(s p l)-C$, the bHLH and the Brd-class. In the fly, $m \alpha$ and bHLH genes are similarly expressed $[14,15,22]$. It is not unlikely that they share common regulatory elements that could explain their co-segregation in the process of evolution.

What about the third bHLH coding gene found in Apis, A.m. $m \beta$ '? This gene may have derived by duplication of A.m. $m \beta$ or of A.m.m $\%$. It is peculiar that this protein is more similar to $D$. melanogaster $\mathrm{M} \beta$ protein than to either A.m.M $\beta$ or A.g.M $\beta$ (see Table 3 ). In contrast, A.m.M $\gamma$ is more similar to A.m.M $\beta$ than to A.m.M $\beta$ '. Furthermore this gene has three introns; one of them is larger than $4 \mathrm{~kb}$ reminiscent of Drosophila hairy or $d p n$ intron sizes. We think that it is unlikely that A.m.m $\beta^{\prime}$ encodes one of the other Hairy/E(spl)-type proteins since respective orthologs were found in the A. mellifera genome with the exception of Her, which seems also absent from the mosquito genome. However, there are similarities between the A.m.M $\beta^{\prime}$ and D. melanogaster Her proteins, including one intron which is at a similar position (Fig. 8B). Although highly speculative, one might conclude that the Drosophila Her gene originally derived from an ancient $E(s p l)$ bHLH type gene. However, this speculation has to be proved or disproved by further investigations. The fact that the positions of the introns of Drosophila $d p n$ and hairy are identical and the introns in A.m.m $m$ and A.m. $m \beta^{\prime}$ are at very similar positions (Fig. 8) supports the notion of a common ancestry of these genes.

\section{The Achaete-Scute complex}

Genes related to achaete or scute have been identified in a large number of species, from hydra [47] to mouse [30], and so we expect these also in the different insects. The $A S-C$ was most intensely studied in various species of Schizophora flies, apart from Drosophila [28,37,48-53]. The number of genes varies between one and four, however, is not strictly correlated with the position in the phylogenetic tree. For example, AS-C of Calliphora vicina contains three genes, whereas other dipteran flies like Drosophila contain four. Two genes are found in the branchiopod crustacean Triops longicaudatus [54] and only one in hydra [47]. In Dipteran flies the expression patterns of the proneural genes are largely varied. This is regulated by positional information through the Iroquois Complex and pannier and in addition by a transcriptional feed-back loop involving AS-C proteins. Eventually, neural precursors are selected by the repressive activity of $\mathrm{E}(\mathrm{spl}) \mathrm{bHLH}$ proteins $[55,56]$. Thereby, location and number of the large bristles on the notum is precisely controlled. The mosquito is covered with rows of large sensory bristle, where number and position varies between individuals [57]. This is in accordance with the fact that there is only one scute-like gene, A.g.ash that is expressed all over the presumptive notum in a modular pattern [37]. Recently it was shown that the Anopheles A.g.ash gene can mimic the endogenous Drosophila genes and that overexpression leads to many ectopic bristles [37].

Albeit the bristle pattern on the notum of different Drosophilids varies slightly, bristle number and position is highly stereotyped [58]. Therefore, it is not surprising to find the AS-C highly conserved within Drosophilids. Yet, the rate of change came unexpected and is quite remarkable outside of the bHLH domain. Compared to $\mathrm{E}(\mathrm{spl})$ bHLH proteins, those encoded by AS-C have a rather low degree of similarity, most notably Ac. In fact, the big flesh fly Calliphora vicina, which like Drosophila belongs to the Schizophora, is totally lacking the ac gene and is covered with bristles [51]. In agreement, we were unable to find $a c$ in Anopheles or Apis, arguing for rapid evolution. The best conservation rate is found in Sc and L'sc suggesting high evolutionary pressure and maybe common ancestry. Not only the bHLH domain, but also two small stretches out- 
A
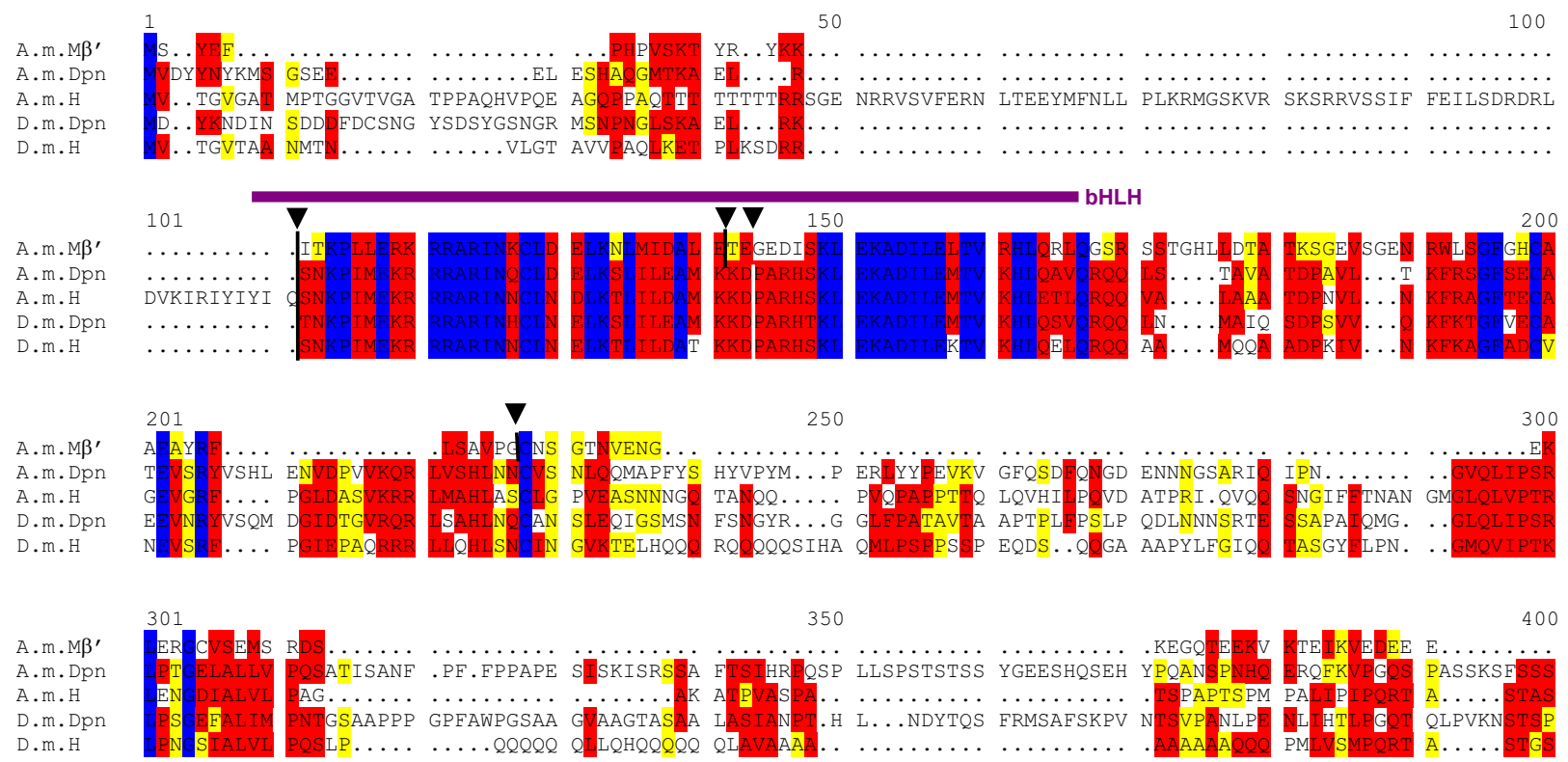

401

A. m. $M \beta^{\prime}$

A.m.Dpn

A.m.H

D.m.Dpn

D.m.H

A.m. $M \beta^{\prime}$

A.m. Dpn

A.m.H

D.m. Dpn

D.m.H
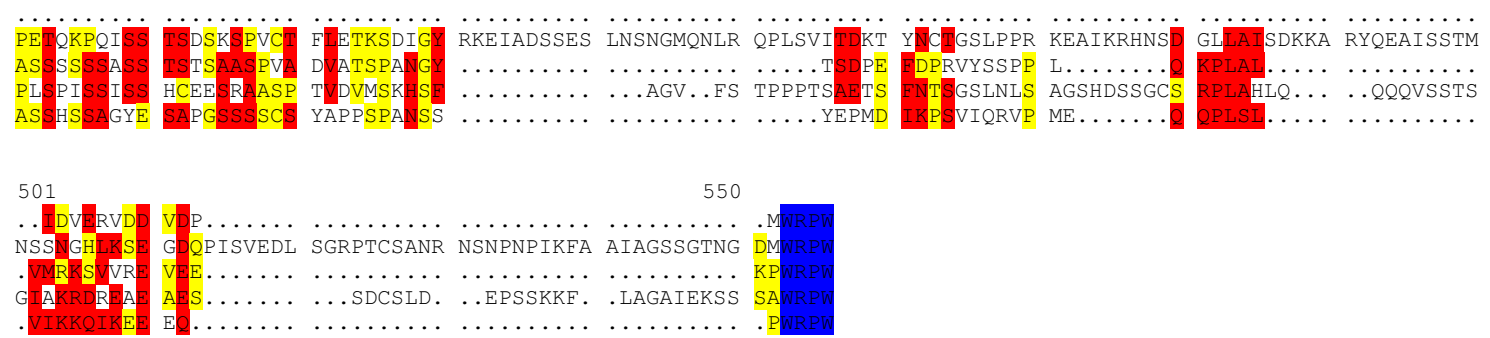

B

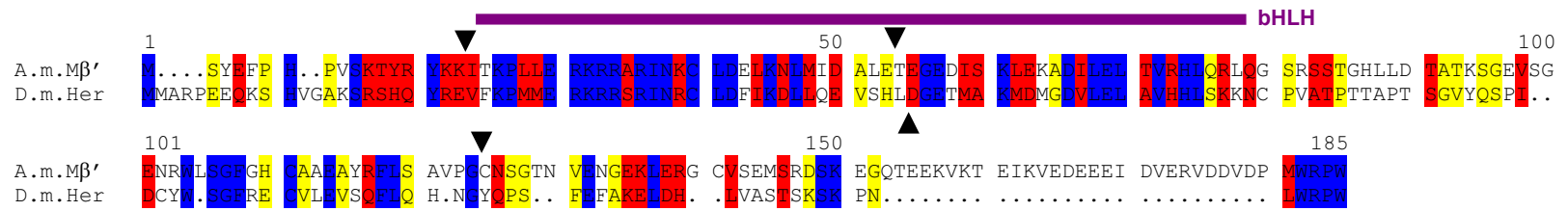

Figure 8

Comparison of A.m.M $\beta$ ' with Dpn, Hairy and Her proteins. A) Comparison of A.m.M $\beta$ ' with A.m.Dpn, A.m.H, D.m.Dpn and D.m.H. The A.m.M $\beta^{\prime}$ protein belongs to the $E(s p l)-C$, however, has several introns (triangle on top, vertical dashes within sequence). Two of the introns are within the bHLH domain at similar position as in D. melanogaster hairy or $d p n$ genes. The bHLH domain is indicated in purple. B) Alignment of D. melanogaster er Hwith A m.M $\beta$ ' protein. Her has only one intron, however, at very similar position as the second intron of A.m.M $\beta$. Introns are marked with triangles. 
Table 3: Comparison between Apis mellifera, D. melanogaster and Anopheles gambia (\% similarity/identity)

\begin{tabular}{|c|c|c|c|c|c|c|}
\hline & A.m.M $\beta$ & A.m.M $\gamma$ & A.m.M $\beta^{\prime \prime}$ & A.m.M $\beta^{\prime 2}$ & D.m. $\mathrm{m} \beta$ & D.m.M $\gamma$ \\
\hline D.m.M $\gamma$ & $72 / 65$ & $72 / 66$ & $71 / 61$ & $71 / 62$ & & \\
\hline D.m.M $\beta$ & $77 / 67$ & $69 / 63$ & $73 / 62$ & $76 / 66$ & & \\
\hline A.g.M $\beta$ & $83 / 76$ & $70 / 66$ & $67 / 57$ & $66 / 57$ & $80 / 70$ & $76 / 67$ \\
\hline A.m.M $\mathrm{M}$ & $73 / 67$ & & $69 / 57$ & $68 / 54$ & & \\
\hline A.m.M & & & $69 / 60$ & $64 / 56$ & & \\
\hline
\end{tabular}

A.m.M $\beta^{\prime \prime}$ : own prediction

A.m.M $\beta^{\prime 2}$ : database prediction

Penalties: bestfit: Gap weight: I, length weight: I, max. penalized length: 30

side (aa 203; SPTPS in D. melanogaster L'sc) and also the C-terminus are of high similarity, the latter found identical in Calliphora [51]. Presumably these protein domains are of functional importance. Indeed, the C-terminus acts as transcriptional activation domain and is also used to recruit $\mathrm{E}(\mathrm{spl}) \mathrm{bHLH}$ proteins [36]. Although the alignments of the respective genes of honeybee and mosquito to $s c$ and $l$ 'sc are very similar, the tendency goes to a closer relationship to l'sc. However, we propose that this gene pair arose by duplication in the course of Drosophilid evolution, such that we may be looking at a common ancestor in the other two species.

The rate of conservation is very limited for the Ase homologs. Decent conservation is found within the bHLH domain, and moreover, a further well-conserved box is present (NGxQYxRIPGTNTxQxL; $x$ are differences between $A$. gambiae and D. melanogaster). This sequence is likewise detected in the Ase protein of C. vicina, which however shares many more similarities with D.m.Ase [51]. In Apis, there is no such conservation outside of the bHLH domain, which itself is highly diverged. The overall degree of conservation is so poor that further statements about the relationship are difficult. We argue that this gene represents A.m.ase by its close proximity to A.m.ash, although other interpretations are similarly possible. An analysis of its expression pattern in honeybee may help to solve these questions.

\section{Conclusion}

We aimed towards an understanding of the evolution of $E(s p l)-C$ and $A S-C$ complexes which in D. melanogaster comprise genes of apparent redundant functions. Our analysis covered insect species that belong to the orders Hymenoptera (honeybee) and Diptera and there to the suborders Nematocera (mosquito) and Brachycera (three species of the genus Drosophila) and thus spans an about 300 Myr window of evolution. We find that both $E(s p l)-C$ and $A S-C$ expanded rather recently as they are only present in their nowadays complex structures in Drosophilids. In Apis and in Anopheles, we find very similar arrangements indicative of an ancient ur-complex. The $E$ (spl)-C seems to have evolved from two genes, one HESlike and one Brd-like gene that are arranged in a head to head orientation. Both types of genes are responsive to Notch signalling in Drosophila. Our data suggest that the most ancient genes are E(spl) bHLH $m \beta$ and $E(s p l) m \alpha$ from which the other $E(s p l)-C$ genes derived by duplication and subsequent change. Moreover, an E(spl) ur-complex is likewise detected in Tribolium castaneum that belongs to the order Coleoptera. In Drosophila the complex also gained unrelated genes like $m 1$ and gro. The latter is highly conserved, however, located at different genomic positions. Whereas in Anopheles the ur-complex seems to exist in its original form, two additional $m \beta$-like bHLH genes are found in the Apis genome that possess introns. These introns are at similar positions as the introns of two other HES-like genes, $d p n$ and $h$ which themselves are highly conserved in the three insect species, arguing for a common evolutionary history. Presumably, the introns are evolutionarily ancient as they are also found in the $C$. elegans $E(s p l) / h$ like gene lin-22. The AS-C seems to originate from a single $s c / l$ 'sc like bHLH gene and a second largely diverged bHLH gene that shares similarity with Drosophila ase. The high degree of variation in the latter makes it difficult to conclusively decide on the original arrangement of this gene complex.

\section{Methods \\ Databases}

Drosophila melanogaster gene and protein sequences were accessed in FlyBase [31]. The D. pseudoobscura database is found at the Human Genome Sequencing Center [59]. The genome of $D$. virilis is sequenced by Agencourt Bioscience Corporation and can be downloaded [60] or searched [61]. The Apis mellifera and Anopheles gambiae databases can be accessed with the Ensembl genome browser [62]. The honeybee genomic sequence is also available at the Human Genome Sequencing Center [59] and the EST sequences were obtained from the honeybee brain EST project [63]. Table 2 lists accession numbers of genes, contig numbers and positions of genes therein as 
well as accession numbers to gene predictions. Due to updates of the databases, gene annotation and names may be different now than reported here.

\section{Sequence analysis}

Sequences were downloaded and further studied applying the HUSAR programs of the Deutsche Krebsforschungszentrum $[64,65]$. Genomic DNA was translated with MAP. BESTFIT and GAP programs were used for alignments and calculation of similarity and identity scores. DOMAIN SWEEP was applied to define bHLH and orange domains, respectively. The amphipathic $\alpha$-helices were predicted and drawn using WHEEL.

\section{D. pseudoobscura sequence acquisition}

The annotation of the D. pseudoobscura database is well advanced. TBlastN searches starting from FlyBase [31] or using D. melanogaster genes allow easy access to both $E(s p l)-C$ and $A S-C$ gene complexes. The database also gives information on transcript length, structure and orientation.

\section{D. virilis sequence acquisition}

Searches were done with BlastN. However, the D. virilis database allows this scan only with DNA against DNA. We therefore searched first for a characteristic part of a D. melanogaster gene (i.e. the bHLH domain) using lowest percent identity option (75\% identity) to find the respective virilis ortholog. We then used the FIND program to identify the respective contig containing these sequences within the downloaded genomic sequence. Subsequently, the entire contig was translated in all six possible reading frames and manually analysed for $E(s p l)-C$ and $A S-C$ genes and proteins. The complete $E(s p l)-C$ was located in one contig. To identify the whole sequence of the AS-C, three overlapping contigs had to be investigated. However, the contigs covering the AS-C still contained many large unsequenced or uncertain stretches. Therefore, the exact size of the complex could not be defined. The sequence gaps do not affect the coding sequences of the studied genes.

\section{A. gambiae and A. mellifera sequence acquisition}

The other databases were screened with $D$. melanogaster protein sequences for members of $E(s p l)-C$ and $A S-C$ against genomic DNA. After detecting convincing similarities, the surrounding genomic DNA was downloaded for further studies with the HUSAR programs. Most of the genes that we describe here have also been annotated as transcribed regions. However, the majority of the database predictions were either incomplete or inconsistent. For our predictions, we carefully analysed genomic DNA and possible coding sequences. All six reading frames were searched for bHLH domain sequences and in the case of the $E(s p l)-C$ bHLH proteins also for WRPW motifs.
Predicted introns were scanned for open reading frames and their borders reinvestigated. Exon/intron boundaries were defined by obeying to the GT AG rule. Afterwards it was analysed whether the newly predicted introns affected the open reading frame.

\section{Classification of gene homology}

Gene homology and orthology was classified based on sequence identity with the respective $D$. melanogaster protein. In the studied Drosophila species all analysed genes have been identified in a 1:1 ratio and could therefore be classified as orthologs. The degree of similarity and identity between two related proteins was determined with the BestFit program [64]. Comparison of proteins from the three Drosophila species was done under the pre-configured standard conditions of the program (Gap weight: 8, length weight: 2). A TBlastN search in the Apis and Anopheles databases always returned several sequences, and we analysed up to 15 of the best hits. If the hit was in a region without predicted gene, we translated the respective sequence and analysed the open reading frames manually. They were analysed for expected protein motifs, like bHLH, orange or WRPW domain. The predicted protein sequences were then used for a BlastP search in FlyBase. In case of a 1:1 relationship, the genes were classified as orthologs. Since there are several E(spl)-C bHLH and AS$C$ bHLH genes in Drosophila, a 1:1 allocation was not possible, therefore, we classified them as homolog (see results). Protein sequences derived from the Apis or Anopheles genome projects are so diverged, that standard conditions only align the best conserved domains. We therefore changed the conditions to Gap weight: 1, length weight: 1 , max. penalized length: 30 . These relaxed conditions had little influence on either alignment of well-conserved sequences, the similarity or identity values. For example, the D.m.M $\beta$ and D.v.M $\beta$ orthologs share $89 \%$ identity under stringent conditions versus 93\% identity under relaxed conditions. The reduced stringency however, allowed an alignment of the entire protein sequence also of the diverged proteins with the consequence that the identity values increased considerably compared to standard conditions. For example an alignment of A.g.Ash (371 residues) with D.m.L'sc (257 residues) under standard conditions aligns only the residues A.g.Ash 50-233 with D.m.L'sc 40-218 with an identity of $46 \%$. Under relaxed conditions the whole protein sequences align with $64 \%$ identity.

\section{Authors' contributions}

RS has contributed substantially to data acquisition and participated in sequence alignments. DM designed the study, acquired and analysed the data and drafted the manuscript. Both authors have read and approved the final manuscript. 


\section{Acknowledgements}

We are indebted to the different Genome Projects for free and easy access to genomic data resources, the FlyBase, the Human Genome Sequencing Center, the Agencourt Bioscience Corporation, the Ensembl Genome Browser and the Honeybee Brain EST Project at the University of Illinois. We thank A. Preiss for constant support and AP, A.C. Nagel and an anonymous reviewer for critically reading the manuscript.

\section{References}

I. Artavanis-Tsakonas S, Rand MR, Lake RJ: Notch signaling: cell fate control and signal integration in development. Science 1999, 284:770-776.

2. Welshons WJ: Dosage sensitive interactions with split mutations in the presence of an Enhancer of split. Drosophila Inf Service 1956, 30: I57-I58.

3. Dawson SR, Turner DL, Weintraub H, Parkhurst SM: Specificity for the hairy/Enhancer of split basic helix-loop-helix (bHLH) proteins maps outside the bHLH domain and suggests two separable modes of transcriptional repression. Mol Cell Biol 1995, | 5:6923-6931.

4. Delidakis C, Artavanis-Tsakonas S: The Enhancer of split locus of Drosophila encodes seven independent helix-loop-helix proteins. Proc Natl Acad Sci USA 1992, 89:873 I-8735.

5. Hartley D, Preiss A, Artavanis-Tsakonas S: A deduced gene product from the Drosophila neurogenic locus, Enhancer of split, shows homology to mammalian G-protein $\beta$ subunit. Cell 1988, 55:785-795.

6. Klämbt C, Knust E, Tietze K, Campos-Ortega JA: Closely related transcripts encoded by the neurogenic gene complex Enhancer of split of Drosophila melanogaster. EMBO J 1989, 8:203-210.

7. Knust E, Tietze K, Campos-Ortega JA: Molecular analysis of the neurogenic locus Enhancer of split of Drosophila melanogaster. EMBO J I 987, 6:4 I I3-4I23.

8. Knust E, Schrons H, Grawe F, Campos-Ortega JA: Seven genes of the Enhancer of split complex of Drosophila melanogaster encode Helix-loop-helix proteins. Genetics 1992, I 32:505-5 I 8.

9. Paroush Z, Finley RL, Kidd T, Wainwright SM, Ingham PW, Brent R, Ish-Horowitz D: groucho is required for Drosophila neurogenesis, segmentation, and sex determination and interacts directly with hairy-related bHLH proteins. Cell 1994 79:805-8I5.

10. Preiss A, Hartley DA, Artavanis-Tsakonas S: The molecular genetics of Enhancer of split, a gene required for embryonic neural development in Drosophila. EMBO J 1988, 7:3917-3927.

II. Eastman DS, Slee R, Skoufos E, Bangalore L, Bray S, Delidakis C: Synergy between Suppressor of Hairless and Notch in regulation of Enhancer of split $\mathbf{m} \gamma$ and $\mathbf{m} \delta$ expression. Mol Cell Biol 1997, I 7:5620-5628.

12. Jennings B, Preiss A, Delidakis C, Bray S: The Notch signaling pathway is required for Enhancer of split bHLH protein expression during neurogenesis in the Drosophila embryo. Development 1994, 120:3537-3548.

13. Jennings B, de Celis J, Delidakis C, Preiss A, Bray S: Role of Notch and achaete-scute complex in the expression of Enhancer of split bHLH proteins. Development 1995, I 2 I:3745-3752.

14. Lai EC, Bodner R, Posakony JW: The Enhancer of split Complex of Drosophila includes four Notch-regulated members of the Bearded family. Development 2000, I 27:344I-3455.

15. Wurmbach E, Wech I, Preiss A: The Enhancer of split complex of Drosophila melanogaster harbors three classes of Notch responsive genes. Mech Dev 1999, 80:171-180.

16. Delidakis C, Preiss A, Hartley DA, Artavanis-Tsakonas S: Two genetically and molecularly distinct functions involved in early neurogenesis reside within the Enhancer of split locus of Drosophila melanogaster. Genetics I99I, I 29:803-823.

17. Nagel AC, Maier D, Krauss S, Mezger M, Preiss A: Neurogenic phenotypes induced by RNA interference with bHLH genes of the Enhancer of split complex of Drosophila melanogaster. Genesis 2004, 39: 105-II4.

18. Schrons H, Knust E, Campos-Ortega JA: The Enhancer of split complex and adjacent genes in the $96 \mathrm{~F}$ region of Drosophila melanogaster are required for segregation of neural and epidermal progenitor cells. Genetics 1992, I 32:48|-503.
19. Cooper MT, Tyler DM, Furriols M, Chalkiadaki A, Delidakis C, Bray S: Spatially restricted factors cooperate with Notch in the regulation of Enhancer of split genes. Dev Biol 2000, 22 I:390-403.

20. de Celis JF, de Celis J, Ligoxygakis P, Preiss A, Delidakis C, Bray S: Functional relationships between Notch, $\mathrm{Su}(\mathrm{H})$ and the bHLH genes of the $E(s p l)$ complex: the $E(s p l)$ genes mediate only a subset of Notch activities during imaginal development. Development 1996, I 22:2719-2728.

21. Tata F, Hartley DA: Inhibition of cell fate in Drosophila by Enhancer of split genes. Mech Dev 1995, 5 I:305-315.

22. Wech I, Bray S, Delidakis C, Preiss A: Distinct expression patterns of different Enhancer of split bHLH genes during Drosophila embryogenesis. Dev Genes Evol 1999, 209:370-375.

23. Maier D, Marte BM, Schäfer W, Yu Y, Preiss A: Drosophila evolution challenges postulated redundancy in the $E(s p l)$ gene complex. Proc Natl Acad Sci USA 1993, 90:5464-5468.

24. Fisher $A L$, Caudy $M$ : Groucho proteins: transcriptional corepressors for specific subsets of DNA-binding transcription factors in vertebrates and invertebrates. Genes Dev 1998 , 12:1931-1940.

25. Fisher $A$, Caudy $M$ : The function of hairy-related bHLH repressor proteins in cell fate decisions. Bioassays 1998, 20:298-306.

26. Artavanis-Tsakonas S, Simpson P: Choosing a cell fate: a view from the Notch locus. Trends Genet 1991, 7:403-8.

27. Campuzano S, Modolell J: Patterning of the Drosophila nervous system: the achaete-scute gene complex. Trends Genet 1992, 8:202-208.

28. Cubas P, de Celis J, Campuzano S, Modolell J: Proneural clusters of achaete-scute expression and the generation of sensory organs in the Drosophila imaginal wing disc. Genes Dev 1991, 5:996-1008.

29. Heitzler P, Bourois M, Ruel L, Cateret C, Simpson P: Genes of the Enhancer of split and achaete-scute complexes are required for a regulatory loop between Notch and Delta during lateral signalling in Drosophila. Development 1996, I22:161-171.

30. Bertrand N, Castro D, Guillemot F: Proneural genes and the specification of neural cell fates. Nature Rev Neurosci 2002, 3:517-530.

31. Flybase, A Database of the Drosophila Genom [http://fly base.bio.indiana.edu/]

32. Gerhart J, Kirschner M: Arthropod phylogeny. In Cells, embryos, and evolution Blackwell Science. Malden; 1997:305.

33. Lai EC, Tam B, Rubin GM: Pervasive regulation of Drosophila Notch target genes by GY-box, Brd-box, and K-box-class microRNAs. Genes Dev 2005, I9:1067-1080.

34. Moore AD, Barbel S, Jan LY, Jan YN: A genomewide survey of basic helix-loop-helix factors in Drosophila. Proc Natl Acad Sci USA 2000, 97: I0436-I044I

35. Prokopenko SN, He Y, Lu Y, Bellen HJ: Mutations affecting the development of the peripheral nervous system in Drosophila: A molecular screen for novel proteins. Genetics 2000, I56:1691-1715.

36. Giagtzoglou N, Koumbanakis KA, Fullard J, Zarifi I, Delidakis C: Role of the Sc C-terminus in transcription activation and $E(s p l)$ repressor recruitment. I Biol Chem 2005, 280: I299-1305.

37. Wülbeck C, Simpson P: The expression of pannier and achaetescute homologues in a mosquito suggests an ancient role of pannier as a selector gene in the regulation of the dorsal body plan. Development 2002, I 29:386 I-387I.

38. Burge C, Karlin S: Prediction of complete gene structures in human genomic DNA. J Mol Biol 1997, 268:78-94.

39. Giagtzoglou N, Alifragis P, Koumbanakis KA, Delidakis C: Two modes of recruitment of $\mathrm{E}(\mathrm{spl})$ repressors onto target genes. Development 2003, I 30:259-270.

40. Gibert J-M, Simpson P: Evolution of cis-regulation of the proneural genes. Int I Dev Biol 2003, 47:643-65I.

41. Massari ME, Murre C: Helix-Loop-Helix proteins: regulators of transcription in eucaryotic organisms. Mol Cell Biol 2000 , 20:429-440.

42. Oellers $N$, Dehio $M$, Knust E: bHLH proteins encoded by the Enhancer of split complex of Drosophila negatively interfere with transcriptional activation mediated by proneural genes. Mol Gen Genet 1994, 244:465-473

43. Lai EC, Posakony JW: Regulation of Drosophila neurogenesis by RNA:RNA duplexes? Cell 1998, 93: I 103-I 104. 
44. Castro B, Barolo S, Bailey AM, Posakony JM: Lateral inhibition in proneural clusters: cis-regulatory logic and default repression by Suppressor of Hairless. Development 2005, 1 32:3333-3344.

45. Carroll SB, Grenier JK, Weatherbee SD: From DNA to diversity: molecular genetics and the evolution of animal design. Volume 46. Blackwell Science. Malden; 200I:I48-I53.

46. McGinnis W, Garber RL, Wirz J, Kuroiwa A, Gehring WJ: A homologous protein-coding sequence in Drosophila homeotic genes and its conservation in other metazoans. Cell 1984 37:403-408.

47. Grens A, Mason E, Marsh JL, Bode HR: Evolutionary conservation of a cell fate specification gene: the Hydra achaete scute homolog has proneural activity in Drosophila. Development 1995, I 21:4027-4035.

48. Alonso MC, Cabrera CV: The achaete-scute gene complex of Drosophila melanogaster comprises four homologous genes. EMBO J I 988, 7:2585-259|.

49. Ghysen A, Dambly-Chaudiere C: From DNA to form: the achaete-scute complex. Genes Dev 1988, 2:495-50I.

50. Gonzalez F, Romani S, Cubas P, Modolell J, Campuzano S: Molecular analysis of the asense gene, a member of the achaete-scute complex of Drosophila melanogaster, and its novel role in optic lobe development. EMBO J 1989, 8:3553-3562.

51. Pistillo D, Skaer N, Simpson P: scute expression in Calliphora vicina reveals an ancestral pattern of longitudinal stripes on the thorax of higher Diptera. Development 2002, I 29:563-572.

52. Skeath JB, Carroll SB: Regulation of the achaete-scute gene expression and sensory organ pattern formation in the Drosophila wing. Genes Dev 1991, 5:984-995.

53. Villares R, Cabrera CV: The achaete-scute gene complex of $D$. melanogaster: conserved domains in a subset of genes required for neurogenesis and their homology to myc. Cell 1987, 50:415-424.

54. Wheeler SR, Skeath JB: The identification and expression of achaete-scute genes in the branchiopod crustacean Triops longicaudatus. Gene Expr Patterns 2005, 5:695-700.

55. Garcia-Garcia MJ, Ramain P, Simpson P, Modolell J: Different contributions of pannier and wingless to the patterning of the dorsal mesothorax of Drosophila. Development 1999 I 26:3523-3532.

56. Gomez-Skarmeta JL, Diez del Corral R, de la Calle-Mustienes E, Ferre-Marco D, Modolell J: Araucan and caupolican, two members of the novel iroquois complex, encode homeoproteins that control proneural and vein-forming genes. Cell 1996, 85:95-105.

57. Simpson $P$, Woehl $R$, Usui K: The development and evolution of bristle patterns in Diptera. Development 1999, I 26: I 349-1 364.

58. Bächli G, Bural H: Taxonomische Merkmale von DrosophilaArten. In Diptera Drosophilidae Schweizerische Entomologische Gesellschaft, Zürich: ETH-Zentrum; 1985:20-30.

59. Human Genome Sequencing Center [http:// www.hgsc.bcm.tmc.edu/]

60. D. virilis (Drosophila virilis) Genome Browser Gateway [http:/ /genome.ucsc.edu/cgi-bin/hgGateway?db=droVirl]

61. NCBI, Trace Archive database Mega BLAST search [http:// www.ncbi.nlm.nih.gov./blast/mmtrace.shtml]

62. Ensembl genome browser [http://www.ensembl.org/]

63. Honeybee brain EST project [http://titan.biotec.uiuc.edu/bee/ honeybee project.htm]

64. HUSAR - Sequence Analysis Package [http://gen ius.embnet.dkfz-heidelberg.de/menu/w2h/w2hdkfz/]

65. Senger M, Flores T, Glatting K-H, Ernst P, Hotz-Wagenblatt A, Suhai S: W2H: WWW interface to the GCG sequence analysis package. Bioinformatics 1998, I4:452-457.
Publish with Biomed Central and every scientist can read your work free of charge

"BioMed Central will be the most significant development for disseminating the results of biomedical research in our lifetime. "

Sir Paul Nurse, Cancer Research UK

Your research papers will be:

- available free of charge to the entire biomedical community

- peer reviewed and published immediately upon acceptance

- cited in PubMed and archived on PubMed Central

- yours - you keep the copyright

Submit your manuscript here:

http://www.biomedcentral.com/info/publishing_adv.asp
BioMedcentral 